Review

\title{
Radiolabeled Nanoparticles for Multimodality Tumor Imaging
}

\author{
Yan Xing ${ }^{1,2}$, Jinhua Zhao ${ }^{2}$, Peter S. Conti ${ }^{1}$, Kai Chen ${ }^{1,}, \mathbb{}$ \\ 1. Molecular Imaging Center, Department of Radiology, Keck School of Medicine, University of Southern California, Los Angeles, CA \\ 90033, USA \\ 2. Department of Nuclear Medicine, Shanghai First People's Hospital, Shanghai Jiao Tong University, Shanghai 200080, China
}

$\square$ Corresponding author: Molecular Imaging Center, Department of Radiology, Keck School of Medicine, University of Southern California, 2250 Alcazar Street, CSC103, Los Angeles, CA 90033, USA. Phone: (323)442-3858; Fax: (323)442-3253; E-mail: chenkai@usc.edu

() Ivyspring International Publisher. This is an open-access article distributed under the terms of the Creative Commons License (http://creativecommons.org/ licenses/by-nc-nd/3.0/). Reproduction is permitted for personal, noncommercial use, provided that the article is in whole, unmodified, and properly cited.

Received: 2013.08.05; Accepted: 2013.10.15; Published: 2014.0I.24

\begin{abstract}
Each imaging modality has its own unique strengths. Multimodality imaging, taking advantages of strengths from two or more imaging modalities, can provide overall structural, functional, and molecular information, offering the prospect of improved diagnostic and therapeutic monitoring abilities. The devices of molecular imaging with multimodality and multifunction are of great value for cancer diagnosis and treatment, and greatly accelerate the development of radionuclide-based multimodal molecular imaging. Radiolabeled nanoparticles bearing intrinsic properties have gained great interest in multimodality tumor imaging over the past decade. Significant breakthrough has been made toward the development of various radiolabeled nanoparticles, which can be used as novel cancer diagnostic tools in multimodality imaging systems. It is expected that quantitative multimodality imaging with multifunctional radiolabeled nanoparticles will afford accurate and precise assessment of biological signatures in cancer in a real-time manner and thus, pave the path towards personalized cancer medicine. This review addresses advantages and challenges in developing multimodality imaging probes by using different types of nanoparticles, and summarizes the recent advances in the applications of radiolabeled nanoparticles for multimodal imaging of tumor. The key issues involved in the translation of radiolabeled nanoparticles to the clinic are also discussed.
\end{abstract}

Key words: radiolabeled nanoparticles; molecular imaging; multimodality imaging; tumor diagnosis; cancer; theranostics

\section{Introduction}

Cancer is the second leading cause of death in the United States and accounts for approximately one in every four deaths. A total of 1,660,290 new cancer cases and 580,350 deaths from cancer are estimated to occur in the United States in 2013 [1]. Despite the fact that rapid advances in molecular biology research have led to an explosion in the knowledge of the molecular bases of cancer over the past few decades, only a minute number of experimental strategies can survive the rigors of clinical trials to become clinically useful. One of major reasons is the lack of tools to directly interrogate the molecular events in cancer patients. The strong impetus to develop noninvasive imaging techniques to visualize molecular changes in patients has brought forth the field of molecular imaging. Molecular imaging can be defined as in vivo visualization, characterization and measurement of biological processes at the molecular and cellular levels $[2,3]$. Up to date, various molecular imaging modalities have been exploited for cancer diagnosis, stratification, and treatment assessment [4]. In contrast to computed tomography $(\mathrm{CT})$, the commonly 
used molecular imaging modalities include positron emission tomography (PET), single photon emission computed tomography (SPECT), molecular magnetic resonance imaging (mMRI), contrast-enhanced ultrasound (CEU), optical bioluminescence, and optical fluorescence [5]. Each imaging modality has its own advantages and limitations as summarized in Table 1. For instance, radionuclide-based imaging techniques, such as PET and SPECT, are highly sensitive and quantitative but they have relatively poor spatial resolution; MRI provides high spatial resolution images with exquisite soft tissue contrast yet it suffers from low sensitivity; optical imaging can sensitively and sequentially interrogate cellular and molecular functions in living subjects, however, the energies in the visible to near-infrared region of the spectrum are limited to penetrate the depth of mammalian tissues. Therefore, combinations of imaging techniques, as so-called "multimodality imaging", are being designed to take advantage the strengths of modalities while minimizing its limitations, which as a result may simultaneously provide comprehensive biological information [6, 7]. The hybrid systems which combine two or more imaging modalities are commercially available, such as PET/CT, SPECT/CT, and PET/MRI, or under active development [8-10].

Table I. Molecular imaging modalities

\begin{tabular}{|c|c|c|c|c|c|c|c|}
\hline Modality & Form of Energy Used & $\begin{array}{l}\text { Spatial Resolu- } \\
\text { tion/mm }\end{array}$ & $\begin{array}{l}\text { Imaging } \\
\text { Time }\end{array}$ & $\begin{array}{l}\text { Required Molec- } \\
\text { ular Probe Mass } \\
\text { (ng) }\end{array}$ & Advantages & $\begin{array}{l}\text { Imaging } \\
\text { Cost }\end{array}$ & $\begin{array}{l}\text { Clinical } \\
\text { Translation }\end{array}$ \\
\hline$\overline{P E T}$ & Annihilation photos & $\begin{array}{l}\text { 1-2 (microPET); } \\
\text { 6-10 (clinical PET) }\end{array}$ & Minutes & $1-100$ & $\begin{array}{l}\text { High sensitivity; } \\
\text { quantitative; tracer } \\
\text { amount of probe }\end{array}$ & High & Yes \\
\hline SPECT & Gamma rays & $\begin{array}{l}\text { 0.5-2 (microSPECT); } \\
\text { 7-15 (clinical SPECT) }\end{array}$ & Minutes & $1-100$ & $\begin{array}{l}\text { High sensitivity; } \\
\text { quantitative; tracer } \\
\text { amount of probe }\end{array}$ & Medium-High & Yes \\
\hline MRI & $\begin{array}{l}\text { Radio frequency } \\
\text { waves }\end{array}$ & $\begin{array}{l}0.01-0.1 \\
\text { (small-animal MRI); } \\
0.5-1.5 \text { (clinical MRI) }\end{array}$ & $\begin{array}{l}\text { Minutes to } \\
\text { hours }\end{array}$ & $10^{3}-10^{6}$ & $\begin{array}{l}\text { High spatial reso- } \\
\text { lution; superb soft } \\
\text { tissue discrimina- } \\
\text { tion }\end{array}$ & High & Yes \\
\hline FI & $\begin{array}{l}\text { Visible to infrared } \\
\text { light }\end{array}$ & $<1$ (FRI); 1 (FMT) & $\begin{array}{l}\text { Seconds to } \\
\text { minutes } \\
\text { (FRI); } \\
\text { minutes } \\
\text { (FMT) }\end{array}$ & $10^{3}-10^{6}$ & $\begin{array}{l}\text { High sensitivity; } \\
\text { multiplexed imag- } \\
\text { ing }\end{array}$ & $\begin{array}{l}\text { Low (FRI); } \\
\text { Medium-High } \\
\text { (FMT) }\end{array}$ & Yes \\
\hline BLI & $\begin{array}{l}\text { Visible to infrared } \\
\text { light }\end{array}$ & $3-5$ & Minutes & $10^{3}-10^{6}$ & $\begin{array}{l}\text { High sensitivity; } \\
\text { high-throughput }\end{array}$ & Low & No \\
\hline US & $\begin{array}{l}\text { High frequency } \\
\text { sound waves }\end{array}$ & $\begin{array}{l}0.04-0.1 \\
\text { (small-animal US); } \\
0.1-1 \text { (clinical US) }\end{array}$ & $\begin{array}{l}\text { Seconds to } \\
\text { Minutes }\end{array}$ & $10^{3}-10^{6}$ & $\begin{array}{l}\text { High sensitivity; } \\
\text { portable }\end{array}$ & Low-Medium & Yes \\
\hline
\end{tabular}

Nanotechnology, the manipulation of matter at the atomic, molecular, and supramolecular scale to create materials with remarkably new properties, is a rapidly expanding area of research with huge potential in many sectors $[11,12]$. In cancer medicine, nanotechnology holds great promise to revolutionize drug delivery, gene therapy, diagnostics, and many areas of research, development and clinical application [13]. Nanoparticles (NPs) extensively employed in nanotechnology are materials with typically overall dimensions less than several hundred nanometers, which are comparable to the size of large biological molecules, such as enzymes, receptors, and antibodies, and about 2-4 orders of magnitude smaller than human cells. Because of this unique physical property, NPs demonstrate marvelous interactions with biomolecules both on the cancer cell surface and inside the cancer cells. For instance, NPs with diameters ranging from 10 to $100 \mathrm{~nm}$ optimally accumulate at the target sites associated with cancer cells due to the enhanced permeability and retention (EPR) effect [14]. Additionally, the large surface area to volume ratio renders NPs with the ability to be readily loaded with a variety of moieties to reach multiple binding sites, suggesting that NPs may provide higher binding efficiencies for targeting specific tumor sites. After labeling with a radionuclide, functionalized NPs can serve as a new class of molecular probes for nuclear imaging. Assuming good stability of the radiolabeled NPs under physiological conditions, the biodistribution of NPs in a living subject can be quantitatively measured by the radiation signals emitted from the radioisotope to noninvasively decipher the biological events, such as tumor receptor levels and tumor enzyme activities. Therefore, cancer imaging using radiolabeled NPs has been attracting great interest in preclinical research and clinical setting. 
In this review, we present an overview of recent developments in the usage of various radiolabeled NPs for multimodality tumor imaging, where the radiolabeled NPs in tumor can be detected by two or more imaging modalities, such as PET/fluorescence, SPECT/fluorescence, PET/MRI, SPECT/MRI, PET/MRI/optical imaging and SPECT/MRI/optical imaging. We also discuss future trends in developing radiolabeled NPs for multimodality tumor imaging.

\section{Radionuclide imaging}

Radionuclide imaging of cancer has been a main emphasis of nuclear medicine research and practice for decades. PET and SPECT are the two major radionuclide imaging modalities in the field of nuclear medicine. Unlike MRI and CT, which mainly provide detailed anatomical images, PET and SPECT can measure chemical changes that occur before macroscopic anatomical signs of a disease are observed. As a revolutionary molecular imaging modality, PET or SPECT traces the in vivo biodistribution of a molecular imaging probe, which is typically labeled with a radioisotope. In PET, the radionuclide decays and the resulting positrons subsequently interact with nearby electrons after travelling a short distance $(\sim 1 \mathrm{~mm})$ within the body. Each positron-electron transmutation produces two 511-keV gamma photons in opposite trajectories, and these two gamma photons may be detected by the detectors surrounding the subject to precisely locate the source of the decay event [15]. Subsequently, the "coincidence events" data can be processed by computers to reconstruct the spatial distribution of the radiotracers. In SPECT, each radionuclide decays from a higher energy state to a lower energy state through the emission of one gamma photon [16]. Detection of photons is performed by a gamma camera comprising a single or multiple detector heads. Unlike in PET, where photons are de- tected in coincidence and can thus be traced back to the same annihilation event, data collection in SPECT is based on the recording of photons detected independently from each other. Single-photon detection relies on the use of physical collimation in order to obtain directional information for the incident photons. In general, SPECT has wider availability than PET, and the radionuclides used for SPECT are readily prepared and usually have a longer half-life than those used for PET. The typical radionuclides for PET and SPECT imaging and the common radiolabeling methods [17-21] are summarized in Table 2.

\section{Nanoparticles for molecular imaging}

Nanoparticles (NPs) can be engineered to target cancer cells for use in the molecular imaging of a malignant lesion. The ultimate goal in the synthesis of multifunctional NPs is the creation of novel NPs for the target-specific diagnosis of cancer. Many types of radiolabeled nanoparticles for multimodality tumor imaging usually have three major components: core, radionuclide, and targeting biomolecule. The targeting biomolecule serves as a carrier for specific delivery of the radionuclide. The radionuclide can be conjugated directly on the surface of nanoparticle core through various labeling methods summarized in Table 2. The radioisotope can also indirectly be attached to the nanoparticle core through a linker. The linker element can be a simple hydrocarbon chain, a peptide, or a polyethylene glycol unit. According to the chemical composition of nanoparticle core, nanoparticles can be broadly classified into inorganic and organic nanoparticles. The typical nano cores used for the construction of radiolabeled nanoparticles are shown in Fig. 1. The existing commercial NPs which can act as contrast agents for multimodality imaging have been summarized in a recent review article [22].

Table 2. Representative radioisotopes and radiolabeling methods for the construction of radiolabeled nanoparticles in cancer imaging

\begin{tabular}{|c|c|c|c|c|c|}
\hline Radioisotopes & Half life & Emission & Energy (KeV) & Radiolabeling Methods & References \\
\hline \multicolumn{6}{|l|}{ PET } \\
\hline${ }^{18} \mathrm{~F}$ & $109.8 \mathrm{~min}$ & $\beta^{+}$ & 634 & $\begin{array}{l}\text { Direct (Nucleophilic or Electrophilic) or Indirect } \\
\text { (Prosthetic) Labeling }\end{array}$ & [17] \\
\hline${ }^{64} \mathrm{Cu}$ & $12.7 \mathrm{~h}$ & $\beta^{-}, \beta^{+}$ & 579,653 & Coordination Chemistry & {$[18]$} \\
\hline${ }^{68} \mathrm{Ga}$ & $67.7 \mathrm{~min}$ & $\beta^{+}$ & 770,1890 & Coordination Chemistry & [19] \\
\hline${ }^{124} \mathrm{I}$ & 4.18 days & $\beta^{+}, \gamma$ & $820,1543,2146$ & Nucleophilic Halogen Exchange Chemistry & {$[20]$} \\
\hline \multicolumn{6}{|c|}{ 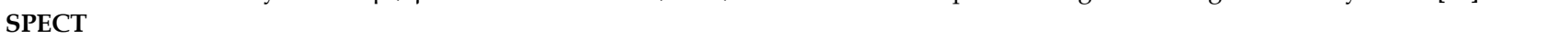 } \\
\hline 99mTc & $6.0 \mathrm{~h}$ & y & 141 & Coordination Chemistry & {$[21]$} \\
\hline${ }^{111}$ In & 2.8 days & $\gamma$, Auger electrons & 172,245 & Coordination Chemistry & [19] \\
\hline${ }^{67} \mathrm{Ga}$ & 3.3 days & $\gamma$ & $93,185,296$ & Coordination Chemistry & [19] \\
\hline
\end{tabular}


Inorganic NPs

Organic NPs

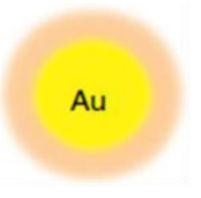

Gold NPs

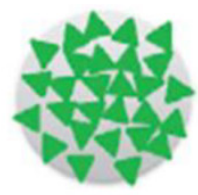

Qdots

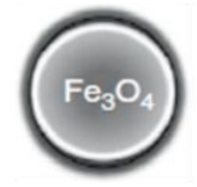
Superparamagnetic
Iron Oxide NPs

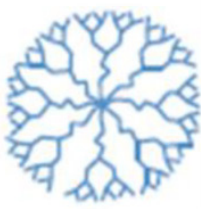

Dendrimers

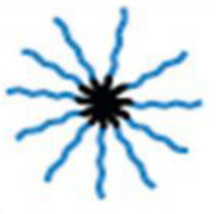

Micelles

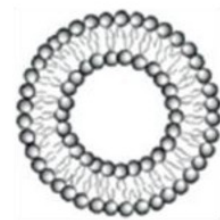

Liposomes

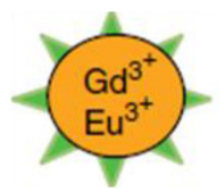

Paramagnetic

Lanthanide Ions

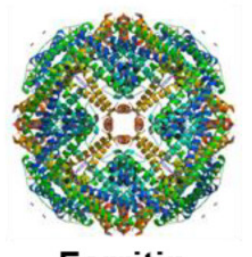

Fig. I. Schematic representation of typical nano cores for the construction of radiolabeled nanoparticles.

Table 3. Representative radiolabeled nanoparticles for multimodality tumor imaging

\begin{tabular}{|c|c|c|c|c|c|}
\hline Classes & Types & $\begin{array}{l}\text { Radio- } \\
\text { nuclides }\end{array}$ & Imaging Methods & Applications & $\begin{array}{l}\text { Refer- } \\
\text { ences }\end{array}$ \\
\hline \multicolumn{6}{|c|}{ Organic NPs } \\
\hline & Ferritin & ${ }^{64} \mathrm{Cu}$ & PET/NIRF & $\begin{array}{l}\text { Integrin } \alpha_{v} \beta_{3} \text {-targeted PET/NIRF imaging with Ferritin nanocages } \\
\text { loaded with RGD peptides, }{ }^{64} \mathrm{Cu} \text {, and Cy5.5 }\end{array}$ & [70] \\
\hline & Micelle & ${ }^{111} \mathrm{In}$ & SPECT/NIRF & $\begin{array}{l}\text { Core cross-linked polymeric micelles conjugated with }{ }^{111} \text { In-labeled } \\
\text { annexin A5 for dual-modality SPECT/NIRF imaging of tumor } \\
\text { apoptosis }\end{array}$ & [71] \\
\hline \multicolumn{6}{|c|}{ Inorganic NPs } \\
\hline & $\begin{array}{l}\text { Quantum } \\
\text { dots }\end{array}$ & ${ }^{64} \mathrm{Cu}$ & PET/NIRF & $\begin{array}{l}\text { Dual-functional targeted delivery with amine functionalized } \\
{ }^{64} \mathrm{Cu} \text {-DOTA-QD-RGD and }{ }^{64} \mathrm{Cu} \text {-DOTA-QD-VEGF for tumor angi- } \\
\text { ogenesis PET/NIRF imaging }\end{array}$ & [66] \\
\hline & Iron oxide & ${ }^{64} \mathrm{Cu}$ & $\mathrm{PET} / \mathrm{MR}$ & $\begin{array}{l}{ }^{64} \mathrm{Cu}-\mathrm{DOTA}-\mathrm{IO}-\mathrm{c}(\mathrm{RGDyK}) \text { for tumor visualization of nude mice } \\
\text { bearing U87MG tumors }\end{array}$ & [73] \\
\hline & Iron oxide & ${ }^{111} \mathrm{In}$ & SPECT/MR & $\begin{array}{l}{ }^{111} \text { In-labeled antimesothelin antibody }\left({ }^{111} \mathrm{In}-\mathrm{mAbMB}\right) \text { with SPIONs } \\
\text { for SPECT/MR imaging of mesothelioma }\end{array}$ & [75] \\
\hline & Iron oxide & ${ }^{64} \mathrm{Cu}$ & PET/MR/Optical & $\begin{array}{l}{ }^{64} \mathrm{Cu} \text {-Cy5.5-HSA-IONPs for tri-modality imaging of nude mice } \\
\text { bearing U87MG tumors }\end{array}$ & [79] \\
\hline & Iron oxide & ${ }^{124} \mathrm{I}$ & PET/MR/Optical & $\begin{array}{l}\text { Cross-linked, superparamagnetic iron oxide nanoparticle } \\
\text { (TCL-SPION) labeled with }{ }^{124} \text { f for PET/MR/optical imaging of } 4 \mathrm{~T} 1 \\
\text { breast tumor }\end{array}$ & [77] \\
\hline & $\begin{array}{l}\text { Lanthanide } \\
\text { nanocrystals }\end{array}$ & ${ }^{124} \mathrm{I}$ & PET/MR/Optical & $\begin{array}{l}\text { 124I-labeled } \mathrm{Er}^{3+} / \mathrm{Yb}^{3+} \text { co-doped NaGdF4 upconversion nanophos- } \\
\text { phors (UCNPs) functionalized with RGD peptide for multimodal } \\
\text { imaging of tumor angiogenesis }\end{array}$ & [81] \\
\hline & Cobalt-ferrite & ${ }^{67} \mathrm{Ga}$ & SPECT/MR/Optical & $\begin{array}{l}{ }^{67} \text { Ga-labeled cobalt-ferrite NPs conjugated with the AS1411 ap- } \\
\text { tamer for multimodal imaging of C6 tumor }\end{array}$ & [82] \\
\hline
\end{tabular}

\section{I. Inorganic nanoparticles}

Inorganic NPs have recently gained significant attention due to their unique material- and size-dependent physicochemical properties, which may not be possible for traditional lipid or polymer based NPs. In particular, characteristics such as chemical inertness, good stability, and ease of functionalization for MRI and optical imaging, make inorganic NPs charming for multimodality imaging of malignant tumor.

\section{I.I. Gold nanoparticles}

Gold nanoparticles (GNPs) are attractive for the construction of imaging agents due to their appealing properties, such as size controllability, good biocompatibility, and easy surface modification [23]. The GNPs also exhibit unique optical properties such as distinctive extinction bands in the visible region because of the surface plasmon oscillation of free electrons [24]. In order to utilize the GNPs as multi-functional probes such as optical quenching, X-ray absorption, and surface enhanced Raman scattering (SERS), the optical and electronic properties of GNPs 
can be readily tunable by changing the size, shape, surface chemistry, or aggregation state. The wet chemical synthesis is usually considered as a major approach to fabricate GNPs through the reduction of gold halides by various chemical reducers.

For medical imaging application, the in vitro toxicology and in vivo biodistribution of GNPs are the major concerns. A wealth of reports suggests that the particle size may be one of key parameters to define the biological characteristic of GNPs. The GNPs within the size range of 10 to $100 \mathrm{~nm}$ are primarily accumulated in all organs of the reticuloendothelial system (RES), and the uniformity of distribution increases with a decrease in particle size [25]. Rapid decrease of the concentration of GNPs in blood and their clearance from the body are related to the functioning of the hepatobiliary system. The GNPs within the size range of 15 to $20 \mathrm{~nm}$ may across the blood-brain or blood-retinal barriers [26]. Small GNPs with diameters of 1 to $2 \mathrm{~nm}$ have potentially high toxicity because of the possibility of irreversible binding to some key biopolymers [27]. In addition, the biodistribution of GNPs over organs and tissues are strongly affected by particle surface modifiers. A well-designed surface modifier can ensure a favorable accumulation contrast in a target organ.

\subsubsection{Quantum dots}

Quantum dots (QDs) are nanometer-sized semiconductor crystals that glow when stimulated by an external source such as ultraviolet light [28]. The wavelength or color of the light emitted from a QD depends on the size of the crystal. Smaller QDs $(\sim 2$ $\mathrm{nm}$ diameter) demonstrate blue fluorescence emission (380 to $440 \mathrm{~nm}$ ), while larger particles ( $\sim 5 \mathrm{~nm}$ diameter) exhibit red fluorescence emission (605 to $630 \mathrm{~nm})$ [29]. The QDs with the sizes $<5 \mathrm{~nm}$ diameter can be rapidly cleared by kidneys in mice [30]. Structurally, QDs consist of a metalloid crystalline core and a "cap" or "shell" that shields the core and renders the QD biocompatible [31]. QDs consist of a variety of metal complexes such as semiconductors, noble metals, and magnetic transition metals (e.g., indium arsenate, gallium arsenate, zinc selenium, cadmium selenium, cadmium tellurium, and lead selenium). QDs must be rendered water-soluble via hydrophilic encapsulation before using for biomedical imaging. Thiol groups are generally anchored on the ZnS shell with terminal carboxyl groups in order to increase the hydrophilicity [32]. Biocompatible coatings or functional groups can be added to the QD's core-shell to improve QD core durability and suspension characteristics, and assign a desired bioactivity, such as drug delivery or molecular imaging. Compromise of the coating may reveal the metalloid core, which may be toxic either as a composite core (e.g., cadmium telluride), or upon dissolution of the QD core to constituent metals (e.g., cadmium) [31]. Compared with organic dyes and fluorescent proteins, QDs have unique optical and electronic properties such as size and composition-tunable fluorescence emission from visible to infrared wavelengths, large absorption coefficients across a wide spectral range, and very high levels of brightness and photostability. Due to the broad wavelength range for excitation, narrow and symmetric emission spectrum properties, QDs are well suited to optical multiplexing in which multiple colors and intensities are combined to encode genes, proteins, and small molecule libraries for imaging tumors [33-35].

\section{I.3. Iron oxide nanoparticles}

The iron oxide (IO) NPs are typically classified by their sizes as standard superparamagnetic iron oxide (SSPIO) at $60-150 \mathrm{~nm}$, ultra small superparamagnetic iron oxide (USPIO) of approximately 5-40 $\mathrm{nm}$, and monocrystalline iron oxide (MION) - a subset of USPIO ranging from 10 to $30 \mathrm{~nm}$. Among these IONPs, the SPIONPs have unique properties such as biocompatibility, intrinsic ability to enhance MR contrast, and facile surface modification, making them attractive as multifunctional nanoplatforms for tumor imaging. The structure of SPIONPs is composed of ferric iron $\left(\mathrm{Fe}^{3+}\right)$ and ferrous iron $\left(\mathrm{Fe}^{2+}\right)$. The SPIONPs can produce predominant T2 relaxation effect, resulting in a signal reduction on T2-weighted MR images. This phenomenon is caused by the magnetic field heterogeneity around the NPs, through which water molecules diffuse, leading to dephasing of the proton magnetic moments [36].

In terms of cytotoxicity, the SPIONPs can be trapped into the RES through endocytosis or phagocytosis. The SPIONPs are considered to be benign to the body because iron oxide is dissolved under acidic conditions [37]. The resulting $\mathrm{Fe}^{3+}$ ions can be fed into the natural iron storage which is $3-5 \mathrm{~g}$ iron for an adult human [38]. Therefore, the additional amount of iron released from dissolved iron oxide NPs is negligible if iron oxide NP concentrations in the $\mu \mathrm{g} \bullet \mathrm{kg}^{-1}$ body weight range are injected [37]. Although some iron oxide NPs exert toxic effects, coated SPIONPs have been found to be relatively nontoxic [39]. As MR contrast agents, SPIONPs are typically coated with sugars such as dextran or synthetic polymers such as silicone [40]. In order to further minimize the toxicity, the IONPs-based MRI contrast agents under current development are usually conjugated with tumor-specific targeting moieties. 


\section{I.4. Rare earth nanoparticles and silica nanoparticles}

Lanthanide materials are excellent building blocks as multimodal imaging probes due to their unique luminescent and magnetic properties. For example, $\mathrm{Gd}^{3+}$ with seven unpaired electrons shows high paramagnetic relaxivity and thus, $\mathrm{Gd}^{3+}$-containing materials can be used as positive $\mathrm{T} 1$ relaxation contrast agents for MRI. In addition, some lanthanide ions codoped NPs show unique upconversion luminescence (UCL). For instance, $\mathrm{NaYF}_{4} \mathrm{NPs}$ doped with $\mathrm{Yb}^{3+}$ and $\mathrm{Er}^{3+}$ display UCL with maxima at $520 \mathrm{~nm}$ and $650 \mathrm{~nm}$, under continuous-wave (CW) excitation at $980 \mathrm{~nm}$ [41-43].

In recent years, silica nanoparticles have received immense interest in cancer imaging because they are chemically inert, optically transparent, and easily modified through chemistry. Co-existence of hydrophilic surface silanol $(-\mathrm{Si}-\mathrm{OH})$ and deprotonated silanol (-Si-O-) groups at neutral $\mathrm{pH}$ makes silica NPs water dispersible, which has the potential to reduce nonspecific binding and in vivo aggregation of silica NPs. After loading fluorescent agents of various kinds, the resulting fluorescent silica NPs are quite photostable [44]. There is an increase in the photostability of fluorescent dyes as they remain protected by the surrounding silica layer. As a result of this protection, photochemical oxidation of fluorescent dyes by the reactive oxygen species is expected to be minimal.

The mesoporous silica materials have achieved great progress in structure control and functionalization (magnetism and luminescence) design. Owing to some unique characteristics, such as large surface area, high pore volume, non-toxicity and good biocompatibility, the mesoporous silica materials have proven to be effective as drug delivery system in biomedical application. Lin and colleagues developed some facile approaches to synthesize multifunctional drug delivery systems which integrated mesoporous, up-conversion luminecent and magnetic properties. For example, $\mathrm{Fe}_{3} \mathrm{O}_{4} @ \mathrm{nSiO}_{2} @ \mathrm{mSiO}_{2} @ \mathrm{NaYF}_{4}: \mathrm{Yb}^{3+}, \mathrm{Er}^{3+}$ nanocomposites or $\mathrm{Gd}_{2} \mathrm{O}_{3}$ : $\mathrm{Eu}^{3+} @ \mathrm{HMS}$ (Hollow Mesoporous Silica) microspheres were successfully used as drug carriers [45-47]. Because these nanoparticles showed up-conversion emission signals or spin-lattice relaxation time $\left(\mathrm{T}_{1}\right)$ weighted effect, they are suitable for the drug delivery systems to monitor the drug release. These mesoporous silica NPs may potentially be used for radionuclide imaging when they are labeled with radioisotopes.

\subsection{Organic nanoparticles}

Over the last decade, a number of organic NPs, such as dendrimers, polymeric micelles, liposomes, and proteins have been used in various applications for diagnosis of cancer. These organic NPs carrying imaging moieties such as radionuclides and NIRF dyes have shown potential for tumor diagnosis.

\subsection{Dendrimers}

Dendrimers are a group of highly branched spherical polymers that are synthesized with structural control rivaling traditional biomolecules such as DNA/RNA, and are often referred to "artificial proteins". Imaging moieties can be encapsulated in the internal cavity or on the surface of the dendrimers with high loading efficiency. By controlling the degree of polymerization, dendrimers can be constructed in various sizes, molecular weights, and chemical compositions. Due to the high loading capacity of payloads and the capability to control the polymer structure, dendrimers are favorable platforms for the construction of multimodal imaging probes [48]. Through the modification of cores, interiors, and surface groups of dendrimer, the properties of dendrimer-based imaging probes can be optimized to reach favorable physical characteristics, biodistribution, receptor-mediated targeting, and controlled release of the payload. Such modifications enable imaging probes to target the areas or organs of interest for facilitating target-specific imaging, as well as assume better excretion pathways that do not interfere with desired applications. By taking advantage of the numerous attachment sites available on the surface of a single dendrimer molecule, various dentrimer-based imaging probes have been built up by new synthetic chemistry strategies for multimodality tumor imaging $[49,50]$.

\subsubsection{Micelles}

Micelles are self-assembling colloidal nanoparticles with a hydrophobic core and hydrophilic shell. They can passively accumulate in the areas with leaky vasculature such as tumors, inflammation, and infarction. In pharmaceutical development, micelles have been successfully used as carriers for water-insoluble drugs [51, 52]. Recently, polymer micelles are gaining an increasing attention for tumor imaging due to their high stability and good biocompatibility. A special group of polymeric micelles can be synthesized by the conjugation of soluble copolymers with lipids (such as polyethylene glycol-phosphatidyl ethanolamine, PEG-PE). The modified micelles can carry various specific targeted moieties on the surface, which may be suitable as imaging probes for multimodal tumor diagnosis.

\subsubsection{Lipsomes}

Liposomes are lipid bilayer nanoparticles enclosing an aqueous compartment that can accommodate lipophilic or hydrophilic drug molecules. Lipo- 
somes have been extensively used for the construction of biocompatible, nontoxic, and biodegradable materials in drug delivery systems. Liposomes can passively accumulate in tumor by extravasation through abnormal leaky tumor vasculature, or actively accumulate by tumor cell specific targeting. Liposomes have versatile modifiability by encapsulating the functional molecules in the inner, inserting in the bilayer, or attaching on the bilayer membrane surface. All these unique properties make liposomes an excellent platform for the specific delivery of imaging moieties. Various methods have been established for preparing liposomes with different sizes, structure, and size distribution [53]. To date, a few liposome formulations have been approved by the FDA for clinical cancer treatment or actively tested under trials [54].

Biodistribution of liposomes is one of major concerns for their application in clinic. Like other nanoparticles, conventional liposomes are vulnerable to eliminate from systemic circulation by the cells of the RES [55]. A number of studies have shown that $50 \% \sim 80 \%$ of liposomes are adsorbed by the RES, primarily by the Kupffer cells of the liver within the first 15-30 $\mathrm{min}$ after intravenous administration [56-58]. It has been found that reducing the size and modulating the surface charge of liposomes could decrease the uptake of RES. As a result, liposomes with small diameter $(80-200 \mathrm{~nm})$ composed of neutral and/or negatively charged lipids and cholesterol have been developed for imaging and therapeutic applications [53].

\subsubsection{Ferritin}

Ferritin is a family of proteins found in different forms in most living organisms. Each ferritin is made of 24 subunits, which self-assemble to form a cage-like nanostructure, with $8 \mathrm{~nm}$ of internal diameter and 12 $\mathrm{nm}$ of external diameter [59]. This unique architecture provides both inside and outside interfaces for possible functional loading [60]. The outer surface of ferritin can be chemically or genetically modified with functional motifs, while the cavity of the ferritin can bind a wide range of metals with high affinities [61-63]. For example, a biovector was conjugated onto ferritin surface, and the resulting NPs were evaluated for targeting C32 melanoma cancer cells [60]. To construct a MRI contrast agent, gadolinium can be loaded into the cavity of ferritin nanocage to target tumor endothelial cells [64]. Most interestingly, it was found that the assembly of ferritin is $\mathrm{pH}$ dependent, despite of its rigidness under physiological condition [65]. The nanoarchitecture can be broken down in an acidic environment and restored, almost intactly, by retuning the $\mathrm{pH}$ value to 7.4. These unique properties make ferritin a powerful nanoplatform on which to construct multifunctional probes for tumor imaging.

\section{Radiolabeled nanoparticles for multi- modality tumor imaging}

Molecular imaging using NPs has been widely applied because of its great potential for early detection, accurate diagnosis, and treatment decision-making in cancer. The development of multimodal probes has been moved forward at an incredibly fast rate by the great progress in the development of hybrid imaging instruments. As multimodal imaging probes, radiolabeled NPs provide several advantages, such as administration of a single contrast agent for different types of imaging modalities, and signal consistency at the target region without the difference in biodistribution that may occur when more than two kinds of contrast agent are used. This section summarizes the recent advances in the development of radiolabeled NPs for multimodality tumor imaging with exemplified studies outlined in Table 3.

\section{I. PET/NIRF imaging}

Near-infrared fluorescence (NIRF) optical imaging offers a noninvasive method for studying diseases at the molecular level in living subjects [5]. As an excellent complement to nuclear imaging techniques, NIRF imaging displays properties of low absorption and relatively low autofluorescence, offering several advantages over other modalities for imaging living organisms. NIRF imaging has high spatial resolution, high sensitivity, and low risk to the living subjects by using nonionizing radiation. PET can be combined with NIRF imaging, allowing increased depth penetration and quantification. The combination of PET and NIRF imaging can provide complimentary information where light penetration is less of an issue.

Cai et al. constructed a novel QD whose surface was successfully conjugated with RGD peptides and $1,4,7,10$-tetraazacyclododecane- $N, N^{\prime}, N^{\prime \prime}, N^{\prime \prime \prime}$-tetraacet ic acid (DOTA) chelators through amide coupling (Fig. 2A) [66]. The resulting QDs (DOTA-QD-RGD) were radiolabeled with ${ }^{64} \mathrm{Cu}$ for PET/NIRF imaging of integrin $\alpha_{\mathrm{v}} \beta_{3}$ expression in tumor-bearing mice. The tumor-targeting efficacy of the dual-functional QD was quantitatively evaluated. In integrin $\alpha_{v} \beta_{3}$ expressing U87MG tumor xenografts, ${ }^{64} \mathrm{Cu}$-DOTA-QD-RGD showed better tumor uptake as compared to ${ }^{64} \mathrm{Cu}-\mathrm{DOTA}-\mathrm{QD}$ at various imaging time points (Fig. 2B). Excellent linear correlation was found between the measurement from PET and NIRF imaging (Fig. 2C). This novel target-specific dual-functional probe significantly reduced potential 
toxicity and overcame the tissue penetration limitation of optical imaging, allowing for quantitative targeted imaging in deep tissue. Later on, a similar study was reported from the same group [67]. Instead of using RGD peptides, the authors adopted the VEGF protein as a moiety for targeting tumor vasculature. Amine-functionalized QD was conjugated with VEGF protein, while a macrocyclic chelating agent - DOTA was used for ${ }^{64} \mathrm{Cu}$ labeling (Fig. 3A). The resulting ${ }^{64} \mathrm{Cu}$-labeled QDs were subject to both PET and NIRF imaging. It was found that U87MG tumor uptake in the mice injected with ${ }^{64} \mathrm{Cu}$-DOTA-QD-VEGF was much higher than that of the mice injected with ${ }^{64} \mathrm{Cu}$-DOTA-QD (Fig. 3B). However, there was a different pattern of tumor signal change between PET and NIRF imaging. The absolute signal intensity from NIRF imaging decreases while PET signal increases overtime. The authors concluded that this observation is likely due to receptor-mediated internalization of ${ }^{64} \mathrm{Cu}$-DOTA-QD-VEGF and trapping of ${ }^{64} \mathrm{Cu}$ inside the tumor by decomposition of ${ }^{64} \mathrm{Cu}$-DOTA complex.
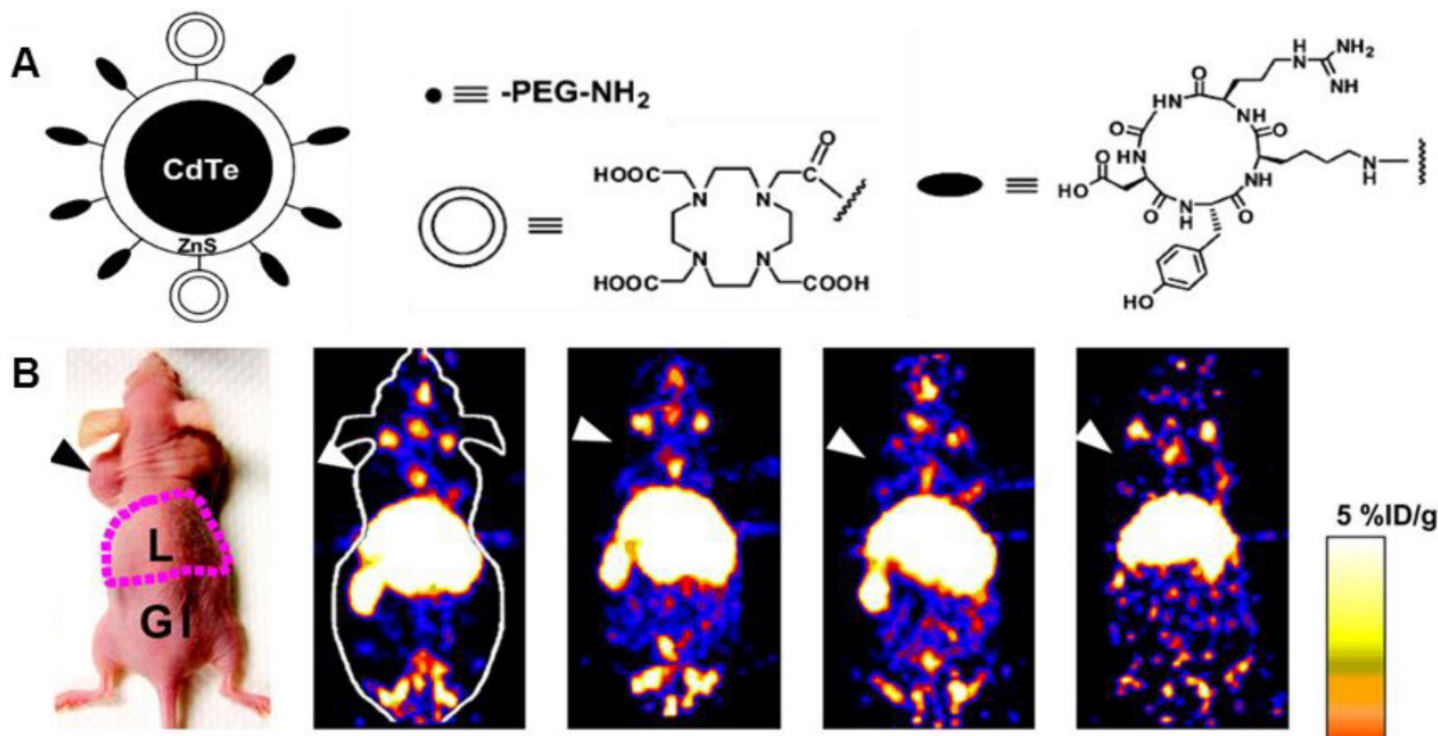

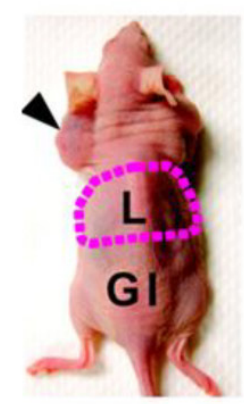

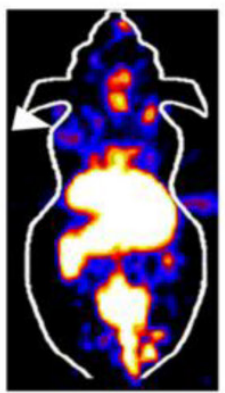

$1 \mathrm{~h}$

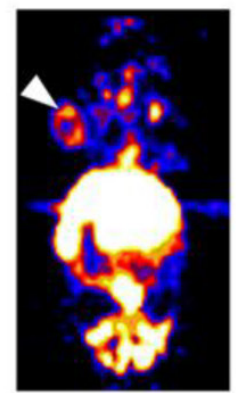

$5 \mathrm{~h}$

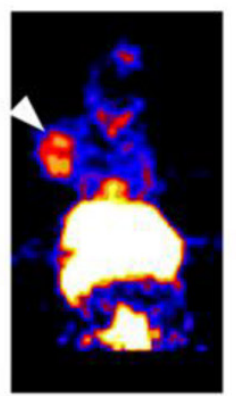

$18 \mathrm{~h}$

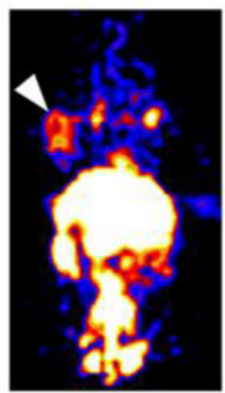

$25 \mathrm{~h}$
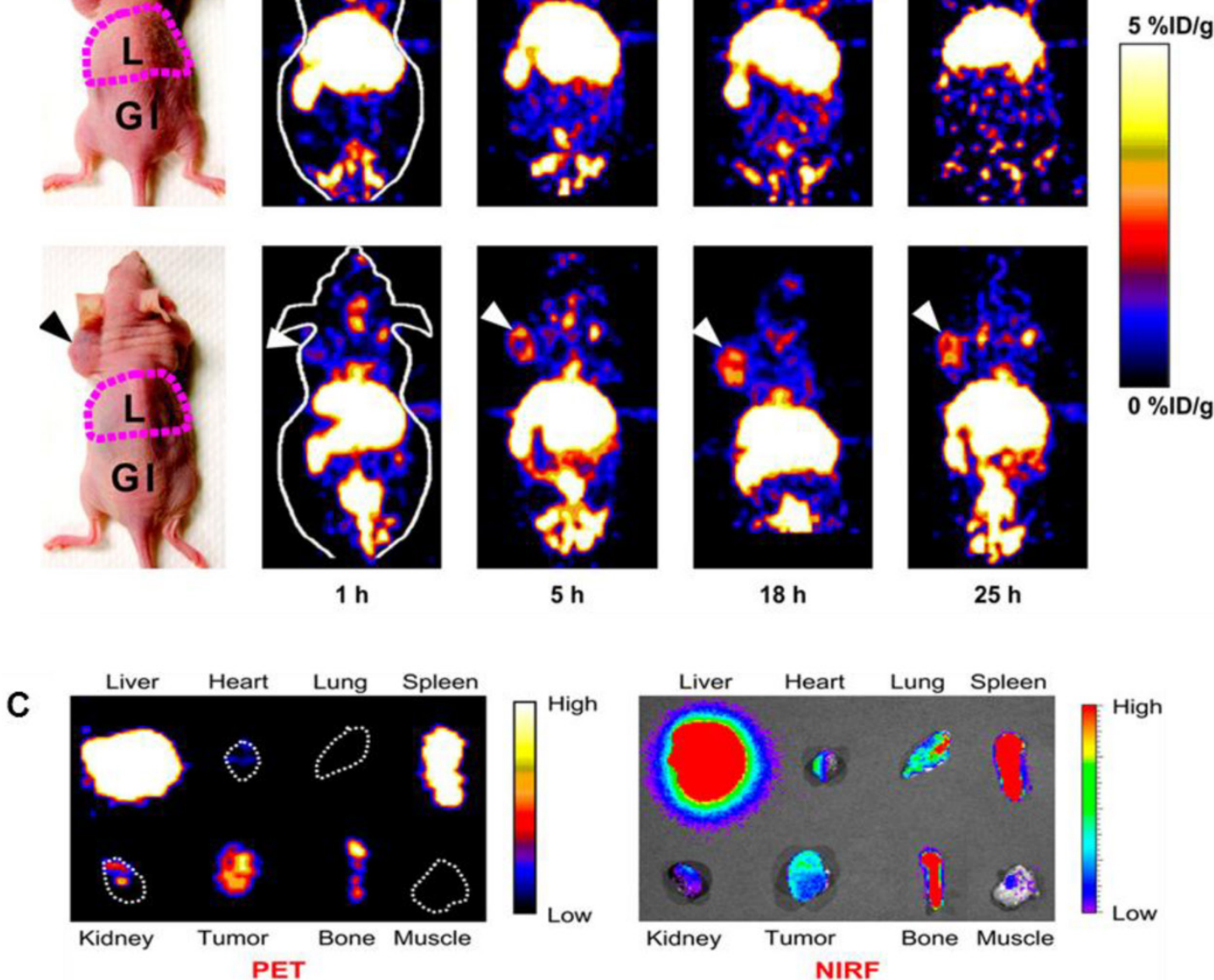

Fig. 2. (A) Schematic illustration of dual-function PET/NIRF probe DOTA-QD-RGD. PEG = polyethylene glycol. (B) Whole-body coronal PET images of mice at I, 5 , 18, and $25 \mathrm{~h}$ after injection of $7-14 \mathrm{MBq}$ of ${ }^{64} \mathrm{Cu}$-labeled DOTA-QD or DOTA-QD-RGD. Arrowheads indicate tumors. Images shown are for slices that were I mm thick. GI = gastrointestinal tract; $\mathrm{L}=$ liver. (C) Left: PET image of harvested tissues at $5 \mathrm{~h}$ after injection of ${ }^{64} \mathrm{Cu}-\mathrm{labeled}$ DOTA-QD-RGD. Right: NIRF image of harvested tissues at $5 \mathrm{~h}$ after injection of ${ }^{4} \mathrm{Cu}$-labeled DOTA-QD or DOTA-QD-RGD. Reprinted with the permission of the Journal of Nuclear Medicine, Cai et al., 2007. 
A

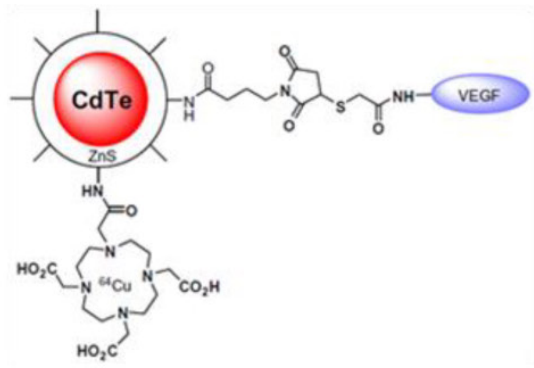

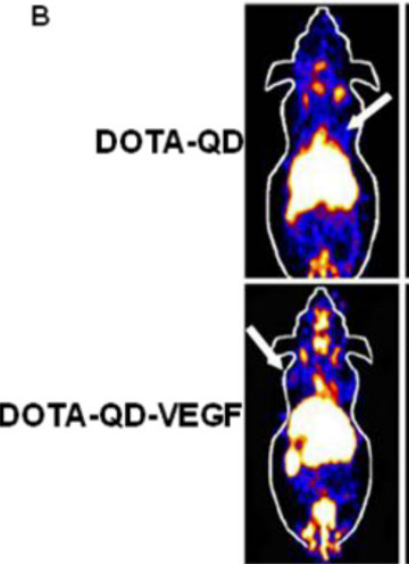

$1 \mathrm{~h}$

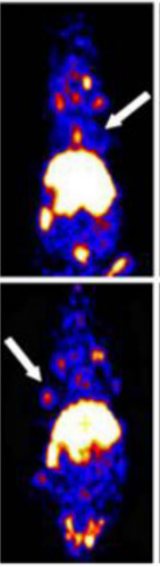

$4 \mathrm{~h}$

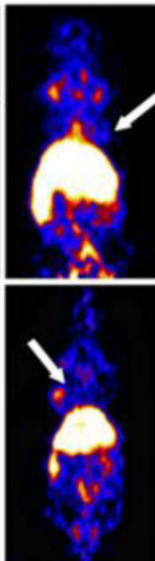

$16 \mathrm{~h}$

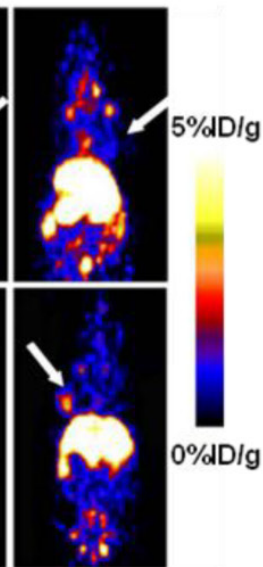

$24 \mathrm{~h}$

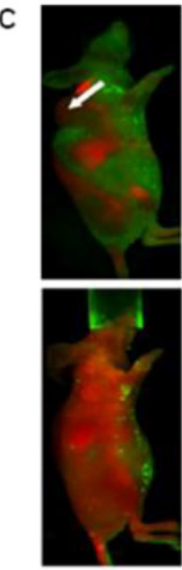

$10 \mathrm{~min}$
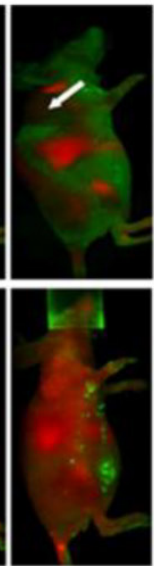

30 min

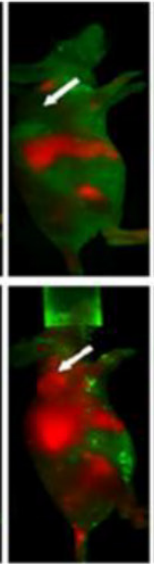

$60 \mathrm{~min}$

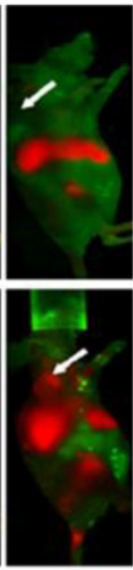

90 min

Fig. 3. (A) Schematic illustration of dual-function PET/NIRF probe DOTA-OD-VEGF. (B) Whole-body coronal PET images of U87MG tumor-bearing mice at I, 4,

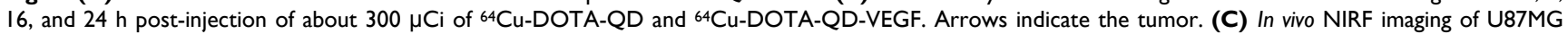
tumor-bearing mice at 10, 30, 60 and 90 min post-injection of 200 pmol of DOTA-QD-VEGF and DOTA-QD, respectively. Arrows indicate the tumor. Reprinted with the permission of the European Journal of Nuclear Medicine and Molecular Imaging, Chen et al., 2008.

Nahrendorf et al. demonstrated the congruence between optical fluorescence-mediated tomography (FMT) and PET measurements using targeted multimodal nanoprobes [68]. Depending on reconstructions of raw datasets, FMT is capable of obtaining quantitative data [69]. Using biocompatible NPs as a platform, the combination of radionuclides, NIR fluorochromes, and target-specific ligands can form multimodal nanoprobes. The study found that there is a high correlation between FMT and PET in nanoprobe concentration and spatial signal distribution both in vitro and in vivo, which justifies the development of next generation of NPs for PET/optical imaging. In another recent study, ferritin nanocages were loaded with RGD peptides, Cy5.5, and ${ }^{64} \mathrm{Cu}$ for integrin $\alpha_{v} \beta_{3}$-targeted PET/NIRF imaging [70]. Unlike traditional conjugation methods, this new loading strategy minimizes the interference among different docked motifs and enables accurate control over the composition of the final conjugates, which provides a very good example for future construction of multimodal NPs-based probes for tumor imaging.

\subsection{SPECT/NIRF imaging}

Another nuclear imaging system which can be combined with optical imaging is SPECT. Similar to PET, SPECT also requires the injection of molecular probes in the tested subject in order to acquire the imaging signal. Instead of detecting the signals from positron-emitting radionuclides for PET, SPECT captures the signals from gamma-emitting radioisotopes. SPECT imaging offers excellent sensitivity but it is limited by relatively poor spatial resolution. In another hand, optical imaging has the potential to offer real-time and high spatial resolution images of tissues, as long as the tissues are accessible with near-infrared light. Importantly, the fluorescent signal from the imaging probes permits ex vivo analysis of excised tissues. A sizable body of research has shown that SPECT/NIRF imaging has great promise in drug development and biomedical studies. An elegant study was performed by Zhang et al. to use polyethylene glycol-coated, core cross-linked polymeric micelles (CCPM) conjugated with ${ }^{111}$ In-labeled annexin A5 for dual-modality SPECT/NIRF tumor imaging [71]. A chelator - diethylenetriaminopentaacetic acid (DTPA) was functionalized on the surface of CCPM for ${ }^{111}$ In labeling through coordination chemistry. The introduction of annexin A5 molecules to CCPM extended the circulation of NPs with a mean half-life of $12.5 \mathrm{~h}$ which was much longer than that of annexin A5. Ex vivo biodistribution studies were performed in tumor-bearing mice with or without chemotherapeutic treatment to induce apoptosis. The radiolabeled NPs, ${ }^{111}$ In-DTPA-A5-CCPM, exhibited significantly 
higher accumulation in the tumors of the treated mice than those of control group. 111In-DTPA-A5-CCPM also showed notably higher tumor uptake in the treated mice than 111In-DTPA-CCPM and 99mTc-labeled hydrazinonicotinamide annexin A5 (99mTc-HYNIC-A5). Autoradiography and immunohistochemistry confirmed that the fluorescence and nuclear signals of ${ }^{111} \mathrm{In}-\mathrm{DTPA}-\mathrm{A} 5-\mathrm{CCPM}$ co-localized with caspase- 3 in the tumor sections from the treated group (Fig. 4). In another example, Liang et al. described the use of streptavidin nanoparticle-based complexes as SPECT/NIRF tumor imaging probes [72]. The NPs consisted of three biotinylated components, including anti-HER2 Herceptin antibody for tumor targeting, DOTA chelator for ${ }^{111} \ln$ radiolabel- ing, and a Cy5.5 fluorophore for NIRF imaging. The components were assembled via streptavidin. The SPECT and NIRF imaging results showed that the resulting ${ }^{111}$ In-DOTA/Cy5.5/Herceptin NPs possess favorable in vivo biodistribution. High tumor accumulation and strong tumor-to-normal tissue contrast can be observed by both SPECT/NIRF imaging. Tumor accumulation of radioactivity at $40 \mathrm{~h}$ can reach 21 ID\%/g, which was much higher than all other tissues, including liver, heart, kidney, spleen, and muscle. The authors summarized that the streptavidin nanoparticle can provide a suitable platform to effectively construct radiolabeled NPs for multimodality imaging of tumor in mice by fluorescence and nuclear detection.

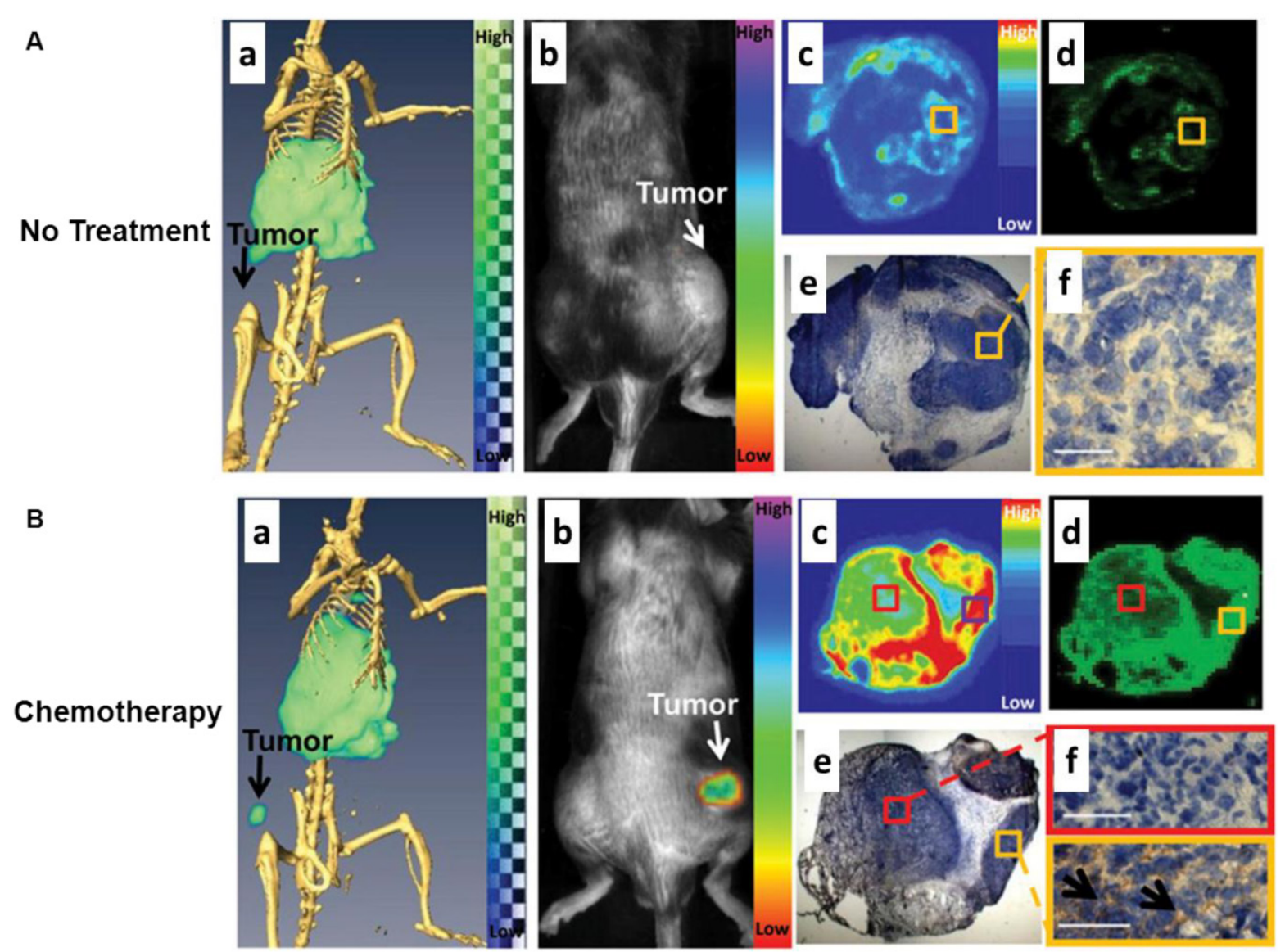

Fig. 4. (A) Control group: dual SPECT/CT and near-infrared fluorescence optical imaging of EL4 lymphoma apoptosis with IIIIn-labeled annexin A5-CCPM. Mice were injected intravenously only with 11 IIn-labeled annexin A5-CCPM. (a) Representative SPECT/CT images. (b) Representative fluorescence molecular tomographic images. (c) Representative autoradiographs of excised tumors. (d) Fluorescence images of same slides used in autoradiographic studies. (e) and (f) Immunohistochemical staining with caspase-3 (brown) of same slides used in autoradiographic studies. All images were acquired at $48 \mathrm{~h}$ after injection of $\mathrm{III}$ In-labeled annexin A5-CCPM. Bar = $50 \mu \mathrm{m}$. (B) Chemotherapy group: dual SPECT/CT and near-infrared fluorescence optical imaging of EL4 lymphoma apoptosis with IIIln-labeled annexin A5-CCPM. Mice in chemotherapy group received intravenous injection of 11 IIn-labeled annexin A5-CCPM $24 \mathrm{~h}$ after treatment with cyclophosphamide (25 $\mathrm{mg} / \mathrm{kg}$ ) by intraperitoneal injection and etoposide $(19 \mathrm{mg} / \mathrm{kg}$ ) by intraperitoneal injection. (a) Representative SPECT/CT images. (b) Representative fluorescence molecular tomographic images. (c) Representative autoradiographs of excised tumors. (d) Fluorescence images of the same slides used in autoradiographic studies. (e) and (f) Immunohistochemical staining with caspase-3 (brown) of the same slides used in autoradiographic studies. All images were acquired $48 \mathrm{~h}$ after injection of $1 \mathrm{II}$ In-labeled annexin A5-CCPM. Bar $=50 \mu \mathrm{m}$. Arrows in (f) refer to the region of tumor apoptosis. Reprinted with the permission of the Journal of Nuclear Medicine, Zhang et al., 20I I. 


\subsection{PET/MR imaging}

MRI is among the best noninvasive imaging techniques used in clinic for assessing anatomy and function of tissues. MRI provides good contrast between the different soft tissues of the body, which makes it especially useful in imaging the brain, the heart, muscles, and tumors compared with other medical imaging techniques such as CT or X-rays. In addition, MRI offers several advantages, such as excellent temporal and spatial resolution and long effective imaging window. However, MRI is less sensitive than PET when used to monitor small tissue lesions, molecular activity, and cellular activities. By the integration of PET and MR, the limitations from each imaging technique can be offset. A very good example is to use an IO-based nanoprobe for simultaneous dual PET and MRI of tumor integrin expression [73]. In this study, RGD peptides were conjugated on the surface of IO NPs where the DOTA chelators were also incorporated for ${ }^{64} \mathrm{Cu}$ labeling. PET/MR imaging was carried out by using ${ }^{64} \mathrm{Cu}$-DOTA-IO-c(RGDyK) NPs to monitor integrin av $\beta 3$ expression levels in U87MG tumor-bearing mice. Tumor uptake of
${ }^{64} \mathrm{Cu}$-DOTA-IO-c(RGDyK) at $1 \mathrm{~h}, 4 \mathrm{~h}$ and $21 \mathrm{~h}$ post-injection was much higher than that of ${ }^{64} \mathrm{Cu}-\mathrm{DOTA}-\mathrm{IO}$, NPs without RGD peptide. Co-injection of a RGD peptide - [c(RGDyK)] with ${ }^{64} \mathrm{Cu}$-DOTA-IO-c(RGDyK) significantly reduced the tumor radioactivity levels, suggesting the target specificity of ${ }^{64} \mathrm{Cu}$-DOTA-IO-c(RGDyK). $\mathrm{T}_{2}$-weighted MRI studies at $3 \mathrm{~T}$ were performed in tumor-bearing mice after intravenous injection of DOTA-IO-c(RGDyK) NPs. At 4 hours post injection, a remarkable tumor signal reduction was observed in the mice receiving DOTA-IO-c(RGDyK) NPs as compared to the results in mice receiving DOTA-IO NPs or co-injection of DOTA-IO-RGDyK (Fig. 5). Staining of iron in tissue sections further confirmed the MRI findings. The success of this PET/MR imaging approach may allow for earlier tumor detection with a high degree of accuracy and provide insight into the molecular mechanisms of cancer with comprehensive information. Except for using ${ }^{64} \mathrm{Cu}$, ${ }^{124} \mathrm{I}$ can be labeled on the surface of IONPs via the tyrosine residue of the albumin coated on the IONPs [74].
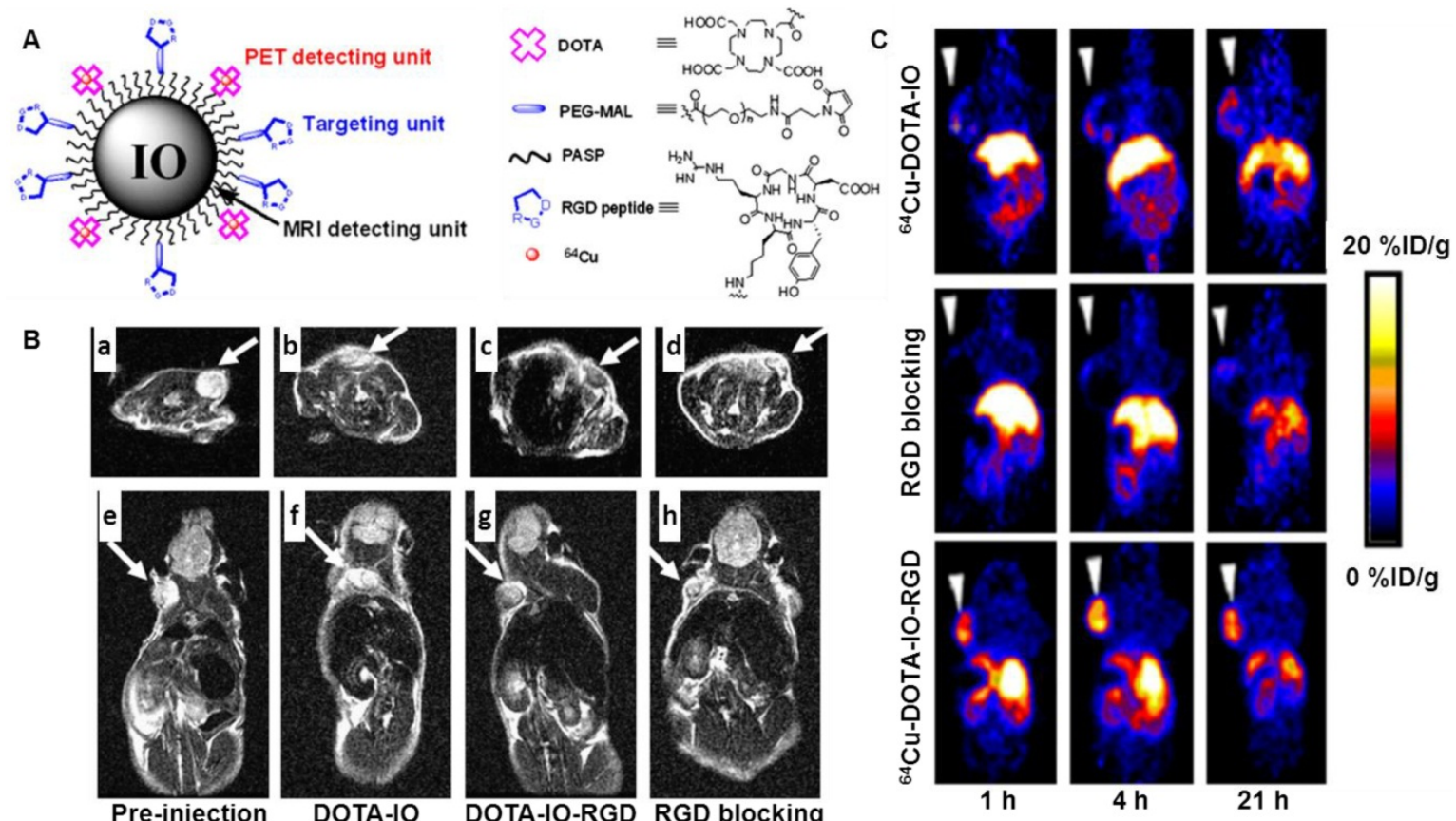

Fig. 5. (A) Schematic illustration of PET/MRI probe based on iron oxide (IO) nanoparticle. (B) T2-weighted MR images of nude mice bearing U87MG tumor before injection of IO nanoparticles ((a) and (e)) and at $4 \mathrm{~h}$ after tail-vein injection of DOTA-IO ((b) and (f)), DOTA-IO-RGD ((c) and (g)), and DOTA-IO-RGD with blocking dose of $c(R G D y K)((d)$ and $(h))$. (C) Decay-corrected whole-body coronal PET images of nude mouse bearing human U87MG tumor at I, 4, and $2 \mathrm{I} h$ after injection of $3.7 \mathrm{MBq}$ of ${ }^{64} \mathrm{Cu}$-DOTA-IO, ${ }^{64} \mathrm{Cu}$-DOTA-IO-RGD, or ${ }^{64} \mathrm{Cu}-\mathrm{DOTA}-\mathrm{IO}-\mathrm{RGD}$ with $10 \mathrm{mg}$ of c(RGDyK) peptide per kilogram (300 $\mu$ g of iron-equivalent IO particles per mouse). Reprinted with the permission of the Journal of Nuclear Medicine, Lee et al., 2008.

\subsection{SPECT/MR imaging}

Specific NPs for SPECT/MR imaging have enormous potential for use in cancer imaging in near-future clinical settings. In an attempt to improve imaging in a few of the most challenging cancers, such as mesothelioma, Misri et al. developed a dual-modality SPECT/MR imaging probe by conjugating ${ }^{111}$ In-labeled antimesothelin antibody (111In-mAbMB) with SPIONs [75]. The use of radio- 
labeled MR probes adds a quantitative component and allows for the determination of the biodistribution and in vivo characteristics of the imaging NPs, which is difficult to achieve solely by MRI. Biodistribution study was carried out to evaluate specific tumor uptake of ${ }^{111} \mathrm{In}-\mathrm{mAbMB}-\mathrm{SPIONs}$ in A431K5 tumors and compare it with nonspecific uptake in A431 tumors. The observed activities in A431K5 tumors were significantly higher $(\mathrm{P}<0.05)$ than in A431 tumors, indicating the specific uptake of ${ }^{111}$ In-mAbMBSPIONs in A431K5 tumors. MRI correlated well with biodistribution findings. The MR signal change was clearly visible in A431K5 tumors between pre- and post-injection time points in the axial MR images, suggesting the presence of iron oxide particles in the tumors. The high relaxivity and specific targeting of ${ }^{111}$ In-mAbMB-SPIONs resulted in an enhanced MR contrast in mesothelin-positive tumors. Overall, ${ }^{111}$ In-mAbMB-SPIONs demonstrated better size distribution and relaxivity properties in comparison with the previously developed MRI and SPECT/MRI contrast agents [76]. It is anticipated that combining SPECT with MR will help obtain both functional and anatomical imaging information with high signal sensitivity and contrast, thereby providing a powerful diagnostic tool for early tumor detection and improved treatment planning.

\subsection{PET/MR/Optical imaging}

Among the various multimodality imaging probes that have been developed to date, hybrid probes for PET/MR/optical imaging have attracted special interest because of the high degree of complementarity among these three imaging modalities [77]. The development of an optical/PET/MR imaging probe promises numerous benefits for pre-clinic research and clinic studies. For example, using a single hybrid probe, the biological events of interest can be rapidly screened with high sensitivity using an easily accessible optical imaging instrument. Subsequently, only the selected objects are subjected to PET and MR imaging for further accurate and quantitative measurement supported by detailed anatomical information. However, integrating multifunctional imaging moieties into a single hybrid probe necessitates a challenge for multistep synthesis and purification process, which often results in significant loss of optical, PET, and MR signals. Therefore, only a few examples of triple-modality imaging probes have been reported so far [78].

Xie et al. modified the surface of IONPs with dopamine to afford nanoconjugates that can be encapsulated into human serum albumin (HSA) matrices [79]. The HSA coated IONPs (HSA-IONPs) were dually labeled with ${ }^{64} \mathrm{Cu}$-DOTA and Cy5.5 to form a hybrid probe, which was tested in a subcutaneous U87MG xenograft mouse model with PET/MR/NIRF imaging (Fig. 6A). For the NIRF imaging, tumor delineation was clearly observed at $1 \mathrm{~h}$ post-injection, and the contrast improved over time (Fig. 6B). The tumor/muscle ratio increased from $1.98 \pm 0.20$ at $1 \mathrm{~h}$ to $2.52 \pm 0.27$ at $4 \mathrm{~h}$, and to $3.08 \pm 0.28$ at $18 \mathrm{~h}(n=$ $3 /$ group). PET imaging showed a gradually elevated tumor uptake at 1, 4, and $18 \mathrm{~h}$ time points (Fig. 6C). Compared with the NIRF imaging results, the PET imaging exhibited higher tumor/muscle ratios of 4.55 $\pm 0.42,5.36 \pm 0.61$, and $8.28 \pm 0.90$ at $1 \mathrm{~h}, 4 \mathrm{~h}$ and $18 \mathrm{~h}$, respectively. Such improvement was presumably due to a lower background in PET. Similar to these observations, MRI scans before the probe injection and $18 \mathrm{~h}$ post particle injection showed a significant signal drop of $29.9 \pm 4.2 \%$ at the tumor sites (Fig. 6D). A clear inhomogeneous particle distribution pattern was observed with MRI, but PET and NIRF results showed homogeneous intensities at the tumor sites. Besides substantial intensities from tumor, accumulation of large amount of probe was also found in the liver. Overall, with the compact HAS coating, the HSA-IONPs manifested a prolonged circulation half-life. Massive accumulation in lesions and high extravasation rate of ${ }^{64} \mathrm{Cu}$-labeled Cy5.5-HSA-IONPs can be observed from PET/MR/optical imaging.

A facile method for the preparation of a PET/MR/optical trimodality imaging probe is to adapt the Cerenkov radiation effect which allows in vivo optical imaging of positron emitting radiotracers. This type of trimodality imaging probe can be constructed by conjugating an appropriate PET radionuclide able to generate Cerenkov light with a magnetic probe. For example, ${ }^{124}$ I can be used as a PET radionuclide [20] due to its sufficiently long half-life (4.176 days) for probe preparation. More importantly, emission of high-energy positrons from ${ }^{124} \mathrm{I}$ during its decay with a $\beta^{+}$mean energy of $819 \mathrm{keV}$ can lead to strong Cerenkov radiation [80]. Park et al. built up a thermally cross-linked, superparamagnetic iron oxide nanoparticle (TCL-SPION) labeled with ${ }^{124} \mathrm{I}$ for PET/MR/optical tumor imaging [77]. Due to the cross-linked polymer coating layers containing polyethylene glycol (PEG) on the magnetite core, the radiolabeled NPs showed high in vivo stability and excellent antibiofouling property. The tumor metastasis model was prepared by injecting the mouse breast cancer cell line 4T1 into the left shoulder of normal BALB/c mice. After ten days, the ${ }^{124}$ I-labeled TCL-SPIONs were injected into both front paws of a 4T1 tumor-bearing BALB/c mouse. Luminescence, microPET and MR imaging was carried out to detect whether the metastasized lymph node could be differentiated from a tumor-free sentinel lymph node 
(SLN) by non-invasive imaging methods. All three imaging modalities consistently showed a much lower uptake of the injected NPs in the left brachial SLN located close to the tumor site, presumably due to the metastasis of the 4T1 tumor cells into the closer lymph node and the destruction of the lymph node function. Among the three imaging modalities, Cerenkov imaging with minimal background and available anatomical information from photographic images exhibited a superior property as compared to the other imaging modalities. Cerenkov imaging does not need any external light source, which minimizes the autofluorescence background typically caused in the case of conventional bioluminescence imaging. PET imaging data were well correlated with the Cerenkov imaging results. Due to the excellent tissue penetration nature in PET, activity distribution in the internal organs can be accurately measured. For MRI, a strong dark spot could be unambiguously assigned to the brachial lymph node on the basis of the detailed tomographic anatomical information. The PET/MR fusion image guided by fiduciary markers revealed the perfect match of the strong blue spot in the PET image with the intense dark spot in the MR imaging. The ex vivo optical and microPET images of the dissected lymph nodes were well matched to the in vivo imaging results. The immunostaining studies of the excised lymph nodes confirmed that the SLN close to the implanted tumor was actually metastasized by 4T1 tumor cells and had a lower uptake of iron-based nanoparticles. This study demonstrated that radiolabeled NPs based on Cerenkov imaging are promising for multimodality tumor imaging.

A

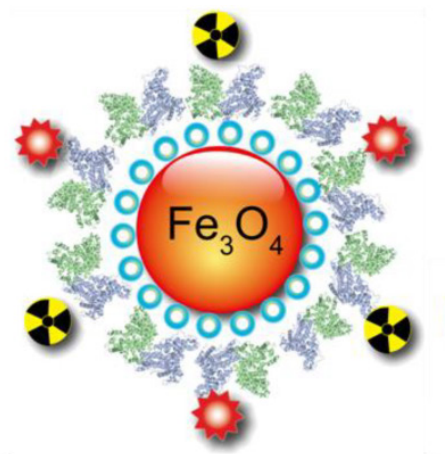

B

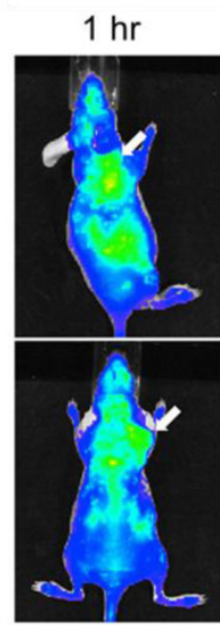

$4 \mathrm{~h}$

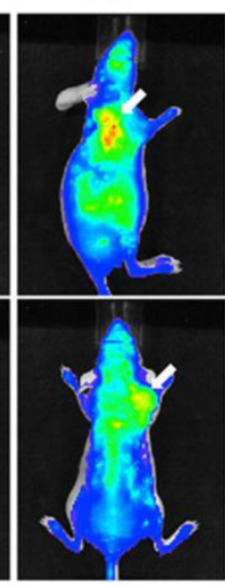

$18 \mathrm{~h}$

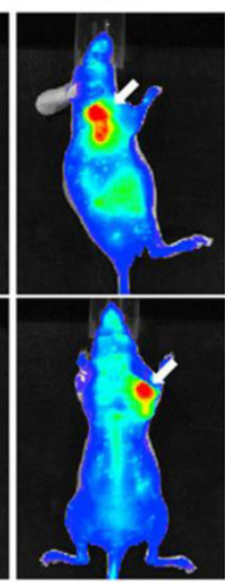

(C) Dopamine

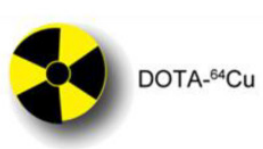

C

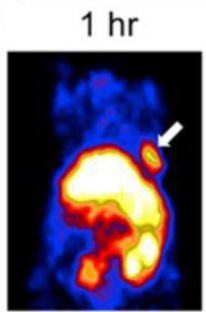

$4 \mathrm{~h}$

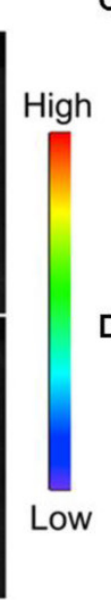

pre-injection

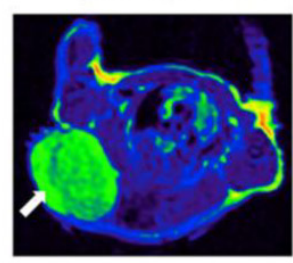

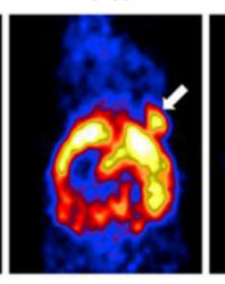

$18 \mathrm{~h}$
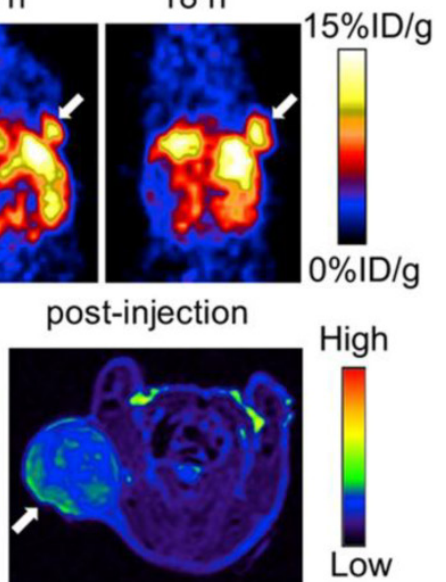

Fig. 6. (A) Schematic illustration of the multi-funtional HSA-IONPs. (B) Representative in vivo NIRF images of mouse injected with HSA-IONPs. Images were acquired I h, $4 \mathrm{~h}$ and $18 \mathrm{~h}$ post injection. (C) In vivo PET imaging results of mouse injected with HSA-IONPs. Images were acquired I h, $4 \mathrm{~h}$ and $18 \mathrm{~h}$ post injection. (D) MRI images acquired before and $18 \mathrm{~h}$ post injection. Reprinted with the permission of the Biomaterials, Xie et al., 2010.

In another study, RGD peptide-functionalized $\mathrm{Er}^{3+} / \mathrm{Yb}^{3+}$ co-doped $\mathrm{NaGdF}_{4}$ upconversion nanophosphors (UCNPs) were radiolabeled with ${ }^{124} \mathrm{I}$, and the resulting radiolabeled NPs were evaluated as a multimodal PET/MR/optical probe for targeting tumor angiogenesis [81]. A dimeric cyclic RGDyK $\left((\mathrm{cRGDyK})_{2}\right)$ peptide was used for the conjugation with polyacrylic acid-coated $\mathrm{NaGdF}_{4}: \mathrm{Er}^{3+} / \mathrm{Yb}^{3+}$
UCNPs and polyethylene glycol molecules. The small-animal PET and clinical 3-T T1-weighted MR imaging of ${ }^{124}$-labeled RGD-functionalized UCNPs were acquired with or without cyclic RGD peptide as a blocking agent in a U87MG tumor model. The results demonstrated that ${ }^{124} \mathrm{I}-\mathrm{c}(\mathrm{RGDyK})_{2}$-UCNPs specifically accumulated in U87MG tumors $(2.8 \pm 0.8$ $\% \mathrm{ID} / \mathrm{g}$ ) whereas the tumor uptake of 
${ }^{124}$ I-c(RGDyK) $)_{2}$-UCNPs was significantly decreased $(1.3 \pm 0.4 \% \mathrm{ID} / \mathrm{g})$ with the blocking RGD peptide, indicating ${ }^{124} \mathrm{I}-\mathrm{C}(\mathrm{RGDyK})_{2}$-UCNPs is a target-specific probe. The T1-weighted MR images showed significant positive contrast enhancement in U87MG tumors. Tumor localization of ${ }^{124} \mathrm{I}-\mathrm{c}(\mathrm{RGDyK})_{2}$-UCNPs was confirmed by inductively coupled plasma mass spectrometry and biologic transmission electron microscopy analysis. Taken together, these results suggest that 124I-labeled RGD-functionalized UCNPs have high specificity for integrin $\alpha_{v} \beta_{3}$-expressing U87MG tumor cells and xenografted tumor models. Multimodal UCNPs can be used as a platform nanoparticle with multimodal imaging for tumor-specific diagnosis.

\subsection{SPECT/MR/Optical imaging}

Hwang et al. reported a tumor imaging probe, using a multimodal cobalt-ferrite NPs conjugated with the AS1411 aptamer for targeting nucleolin protein which is highly expressed on the membrane of cancer cells [82]. The NPs surrounded by fluorescent Rhodamine within a silica shell matrix were coupled with the AS1411 aptamer (MF-AS1411). The NPs were also bound with a chelating agent 2-(p-isothio-cyanatobenzyl)-1,4,7-triazacyclonane-1,4, 7 -triacetic acid ( $p$-SCN-Bn-NOTA) for ${ }^{67} \mathrm{Ga}$ labeling. The purified MFR-AS1411 NPs showed specific fluorescence signals in nucleolin-expressing C6 cells in comparison with MFR-AS1411 mutant MFR-AS1411-treated C6 cells. By increasing the concentration of MFR-AS1411, both the Rhodamine fluorescence intensity and ${ }^{67} \mathrm{Ga}$ activity of MFR-AS1411 were enhanced in a dose-dependent manner. As shown in Fig. 7, the radioactivities emitting from ${ }^{67} \mathrm{Ga}$ can be detected in both thighs of the mice injected with MFR-AS1411, whereas the MFR-AS1411 mutant (MFR-AS1411mt) administration revealed rapid clearance via the bloodstream, indicating that MFR-AS1411 specifically accumulated in the tumor. The results from in vivo MRI and ex vivo optical imaging matched well with SPECT imaging. MR images of tumor-bearing mice before and after injection of MFR-AS1411 were acquired. Significantly higher signal intensities at tumor sites were detected in MFR-AS1411-injected mice as compared to those without the administration of MFR-AS1411.
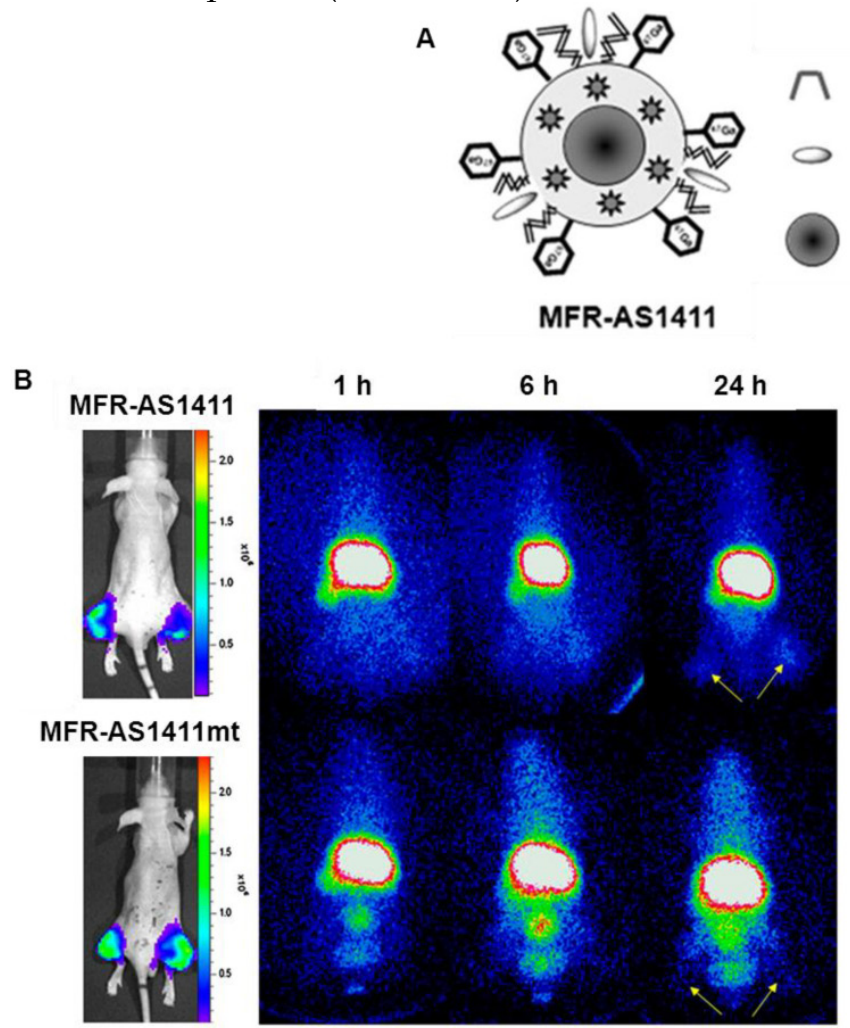
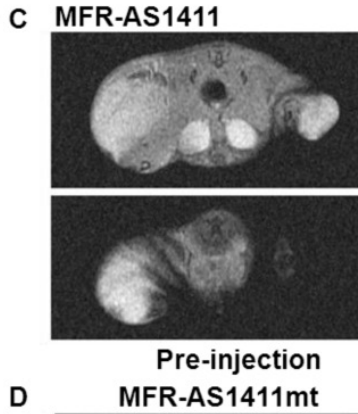

D

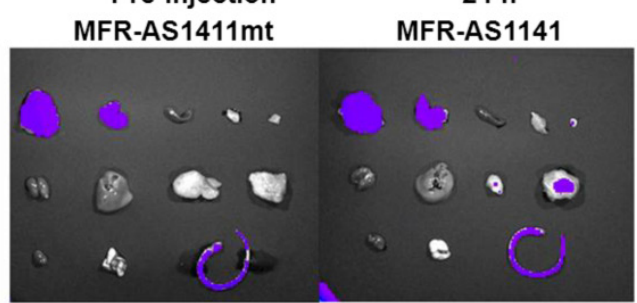

Fig. 7. (A) Schematic illustration of MFR-ASI4II, a cobalt-ferrite nanoparticle surrounded by fluorescent rhodamine (designated MFR) within a silica shell matrix with the ASI4I I aptamer. (B) MFR-ASI4I I particles were intravenously injected into tumor-bearing mice, and radionuclide images were acquired at I, 6 , and $24 \mathrm{~h}$ after injection. Scintigraphic images of C6 tumors in mice that received MFR-ASI 4 I I showed that C6 tumors had accumulated MFR-ASI 4 I I at 24 h after injection but did not accumulate MFR-ASI4II mt $(n=3)$. Tumor growth patterns were followed using bioluminescence signals acquired from luciferase-expressing C6 cells. (C) $M R$ images of tumor-bearing mice before and after injection of MFR-ASI4II were acquired. Dark signal intensities at tumor sites were detected in MFR-ASI4II-injected mice (arrowhead). (D) Fluorescence imaging of isolated organs. Fluorescence signal at tumor site injected with MFRASI4II was detected, compared with tumors injected with MFR-ASI $4 \mathrm{I} I \mathrm{mt}$. Isolated organs in order from upper left to lower right were intestine, liver, spleen, muscle, fat, kidney, stomach, right tumor, left tumor, heart, lung, and tail. Reprinted with the permission of the Journal of Nuclear Medicine, Hwang et al., 2010. 
Optical imaging of dissected organs was carried out to confirm the tumor uptake of MFR-AS1411. The fluorescence signal at tumor site injected with MFR-AS1411 was detected, whereas no fluorescence signal can be observed in the tumors injected with MFR-AS1411mt. The results demonstrated that the favorable tumor targeting of MFR-AS1411 NPs can be monitored by SEPCT/MR/optical imaging both in vitro and in vivo. This study provides a good example that multifunctional imaging modality platforms based on a combination of nanotechnology and molecular imaging has the potential to help us better understand molecular behaviors of tumors.

\section{Conclusion and perspectives}

The idea of applying multiple modalities in a single imaging session comes from the fact that modalities with high sensitivity have relatively poor resolution, while those with high resolution have relatively poor sensitivity $[83,84]$. Development of hybrid imaging technology has triggered great effort in probe development to boost the benefits of hybrid instrument technology [85-87]. Therefore, a variety of NPs have been developed to allow for non-invasive imaging of tumors and exploit the targetable characteristics of cancer [88].

Although there has been an explosion in the application of radiolabeled NPs in multimodality tumor imaging over the past decade, most of studies are proof-of-principle and more research efforts are needed to translate radiolabeled NPs to the clinical stage in the near future. Several key issues need to be addressed for the development of radiolabeled NPs. First of all, the uptake of radiolabeled NPs in the RES organs, such as liver and spleen, is a major challenge for in vivo imaging. The physicochemical properties such as size, hydrophobicity, flexibility, and surface charge of NPs will affect in vivo biodistribution and clearance. Rigid and globular-structured NPs with a size below $6 \mathrm{~nm}$ can be excreted through the renal route. In particular, NPs with 4-8 nm size are rapidly removed from the blood by the RES, leading to rapid uptake in the liver and spleen, and excretion through the hepatobiliary route [30]. Thus, relatively smaller NPs would have lower RES uptake, which can result in better imaging properties. In addition, the dissociation of the radionuclide (typically metal ion) from the chelator, or the radionuclide-containing polymer coating from the NP, may cause significant uptake in normal organs and reduce the specificity of the radiolabeled NPs. A complex of radionuclide-chelator with better in vivo stability needs to be considered [89, 90]. Careful in vitro experiments for measuring the stability of radiolabeled NPs are generally required prior to in vivo studies. Moreover, in order to increase the specific binding of radiolabeled NPs to tumor site, the targeting moieties, such as peptides and antibodies should be prudently chosen, and the physicochemical properties of NPs, such as the size and surface charges, should be extensively explored. Furthermore, cellular toxicity of NPs should be seriously considered in the design of radiolabeled NPs for multimodality tumor imaging. NPs with high cellular toxicity have no chance for clinical study. Some inorganic NPs fabricated with metal elements, such as gadolinium, cadmium (e.g. quantum dots), are known to be toxic [91]. Certain carbon-based NPs also show the biological toxicity [92]. Before NPs can be administrated into the human body for tumor diagnosis, comprehensive assessments in the potential toxicity of NPs should be carefully investigated. In general, biocompatible materials, especially natural materials from the human body, may have higher priority over heavy metals for human use.

As mentioned earlier, the development of radiolabeled NPs is not trivial. The advanced chemistry techniques will likely continue to play an important role in the design and development of radiolabeled NPs for multimodality tumor imaging. For example, "click chemistry" $[93,94]$ offers a platform for general, modular, and high yielding synthetic transformations for constructing highly diverse molecules. Because click chemistry is a modular approach, it is possible to fine tune the pharmacokinetic and pharmacodynamics profiles of imaging probes. Click chemistry has proven itself to be superior in satisfying many criteria, e.g., biocompatibility, selectivity, yield, and stereospecificity. With recent development of click chemistry, significant improvement has been made in a catalyst-free variant of azide-alkyne click chemistry. In this reaction, the release of ring strain in a cycloalkyne precursor lowers the activation energy for the cycloaddition process, enabling the ligation to be performed in high efficiency without a catalyst $[95,96]$. It is expected that click chemistry will become a more routine strategy in the near future for a wide range of applications for the construction of radiolabeled NPs.

It is worthy to mention that radiolabeled NPs can be designed and used not only for tumor diagnosis, but also for therapeutic agents, as so-called "theranostics" [97]. Current progress in nanomedicine has exploited the possibility of designing tumor-targeted NPs to deliver radionuclide in a specific manner to improve the efficacy and safety of cancer imaging and therapy. Radiolabeled NPs after tagging with appropriate chemotherapeutics can gain the ability to image and monitor the tumors, delivery kinetics, and drug efficacy with the long-term hope of optimizing treatment protocols with improved therapeutic efficacy and reduced toxicity to normal tis- 
sues. We can envision that radiolabeled NPs-based theranostic agents will play major roles in cancer diagnosis, early stage anti-cancer drug development, as well as cancer treatment plan in the near future.

In conclusion, it is critical to develop creative strategies that can be used to identify cancer with high sensitivity and high predictive value. Radiolabeled NPs have shown great promise as molecular probes in multimodal tumor imaging. We expect that quantitative multimodality imaging with novel radiolabeled NPs will afford accurate and precise assessment of biological signatures in cancer in a real-time manner and thus, pave the path towards personalized cancer medicine.

\section{Abbreviations}

PET, positron emission tomography; SPECT, single photon emission computed tomography; CT, computed tomography; MRI, magnetic resonance imaging; FI, fluorescence imaging; NIRF, near infrared fluorescence; FRI, fluorescence reflectance imaging; FMT, fluorescence molecular tomography; BLI, bioluminescence imaging; US, ultrasound; CEU, contrast-enhanced ultrasound; NPs, nanoparticles; EPR, enhanced permeability and retention; GNPs, gold nanoparticles; SERS, surface enhanced Raman scattering; RES, reticuloendothelial system; QDs, quantum dots; IO, iron oxide; SPIONPs, superparamagnetic iron oxide nanoparticles; USPIO, ultra small superparamagnetic iron oxide; $\mathrm{PE}$, phosphatidyl ethanolamine; RGD, Arg-Gly-Asp; VEGF, vascular endothelial growth factor; CCPM, core cross-linked polymeric micelles; HAS, human serum albumin; TCL, thermally cross-linked; SLN, sentinel lymph node; UCNPs, upconversion nanophosphors; ID/g, injected dose per gram of tissue.

\section{Acknowledgments}

This work was supported by the research funds from the American Cancer Society (\#IRG-58-007-51), the Robert E. and May R. Wright Foundation, the USC Department of Radiology, and the Natural Science Foundation of China (Grant No. 81171368).

\section{Competing Interests}

The authors have declared that no competing interest exists.

\section{References}

1. Siegel R, Naishadham D, Jemal A. Cancer statistics, 2013. CA Cancer J Clin. 2013; 63: 11-30.

2. Weissleder R, Mahmood U. Molecular imaging. Radiology. 2001; 219: 316-33.

3. Chen K, Chen X. Design and development of molecular imaging probes. Curr Top Med Chem. 2010; 10: 1227-36.

4. Cai W, Chen X. Multimodality molecular imaging of tumor angiogenesis. J Nucl Med. 2008; 49 Suppl 2: 113S-28S

5. Massoud TF, Gambhir SS. Molecular imaging in living subjects: seeing fundamental biological processes in a new light. Genes Dev. 2003; 17: 545-80.
6. Cherry SR. Multimodality imaging: beyond PET/CT and SPECT/CT. Semin Nucl Med. 2009; 39: 348-53.

7. Patel CN, Goldstone AR, Chowdhury FU, Scarsbrook AF. FDG PET/CT in oncology: "raising the bar". Clin Radiol. 2010; 65: 522-35.

8. Beyer T, Townsend DW, Brun T, Kinahan PE, Charron M, Roddy R, et al. A combined PET/CT scanner for clinical oncology. J Nucl Med. 2000; 41: 1369-79.

9. Even-Sapir E, Lerman H, Lievshitz G, Khafif A, Fliss DM, Schwartz A, et al. Lymphoscintigraphy for sentinel node mapping using a hybrid SPECT/CT system. J Nucl Med. 2003; 44: 1413-20.

10. Catana C, Wu Y, Judenhofer MS, Qi J, Pichler BJ, Cherry SR. Simultaneous acquisition of multislice PET and MR images: initial results with a MR-compatible PET scanner. J Nucl Med. 2006; 47: 1968-76.

11. Ferrari M. Cancer nanotechnology: opportunities and challenges. Nat Rev Cancer. 2005; 5: 161-71.

12. Sahoo SK, Parveen S, Panda JJ. The present and future of nanotechnology in human health care. Nanomedicine. 2007; 3: 20-31.

13. Hong H, Zhang Y, Sun J, Cai W. Molecular imaging and therapy of cancer with radiolabeled nanoparticles. Nano Today. 2009; 4: 399-413.

14. Greish K. Enhanced permeability and retention (EPR) effect for anticancer nanomedicine drug targeting. Methods Mol Biol. 2010; 624: 25-37.

15. Chen K, Conti PS. Target-specific delivery of peptide-based probes for PET imaging. Adv Drug Deliv Rev. 2010; 62: 1005-22.

16. Gullberg GT, Reutter BW, Sitek A, Maltz JS, Budinger TF. Dynamic single photon emission computed tomography--basic principles and cardiac applications. Phys Med Biol. 2010; 55: R111-91.

17. Miller PW, Long NJ, Vilar R, Gee AD. Synthesis of ${ }^{11} \mathrm{C},{ }^{18} \mathrm{~F},{ }^{15} \mathrm{O}$, and ${ }^{13} \mathrm{~N}$ radiolabels for positron emission tomography. Angew Chem Int Ed Engl. 2008; 47: 8998-9033.

18. Shokeen M, Anderson CJ. Molecular imaging of cancer with copper-64 radiopharmaceuticals and positron emission tomography (PET). Acc Chem Res. 2009; 42: 832-41.

19. Wadas TJ, Wong EH, Weisman GR, Anderson CJ. Coordinating radiometals of copper, gallium, indium, yttrium, and zirconium for PET and SPECT imaging of disease. Chem Rev. 2010; 110: 2858-902.

20. Holland JP, Williamson MJ, Lewis JS. Unconventional nuclides for radiopharmaceuticals. Mol Imaging. 2010; 9: 1-20.

21. Liu S, Edwards DS. 99mTc-Labeled Small Peptides as Diagnostic Radiopharmaceuticals. Chem Rev. 1999; 99: 2235-68.

22. Wang $\mathrm{Y}, \mathrm{Xu} \mathrm{C}, \mathrm{Ow} \mathrm{H}$. Commercial nanoparticles for stem cell labeling and tracking. Theranostics. 2013; 3: 544-60.

23. Lee DE, Koo H, Sun IC, Ryu JH, Kim K, Kwon IC. Multifunctional nanoparticles for multimodal imaging and theragnosis. Chem Soc Rev. 2012; 41: 2656-72.

24. El-Sayed MA. Some interesting properties of metals confined in time and nanometer space of different shapes. Acc Chem Res. 2001; 34: 257-64.

25. Dykman L, Khlebtsov N. Gold nanoparticles in biomedical applications: recent advances and perspectives. Chem Soc Rev. 2012; 41: 2256-82.

26. Khlebtsov N, Bogatyrev V, Dykman L, Khlebtsov B, Staroverov S, Shirokov A, et al. Analytical and theranostic applications of gold nanoparticles and multifunctional nanocomposites. Theranostics. 2013; 3: 167-80.

27. Khlebtsov N, Dykman L. Biodistribution and toxicity of engineered gold nanoparticles: a review of in vitro and in vivo studies. Chem Soc Rev. 2011; 40: 1647-71.

28. Cheki M, Moslehi M, Assadi M. Marvelous applications of quantum dots. Eur Rev Med Pharmaco. 2013; 17: 1141-8.

29. Bruchez M, Jr., Moronne M, Gin P, Weiss S, Alivisatos AP. Semiconductor nanocrystals as fluorescent biological labels. Science. 1998; 281: 2013-6.

30. Choi HS, Liu W, Misra P, Tanaka E, Zimmer JP, Itty Ipe B, et al. Renal clearance of quantum dots. Nat Biotechnol. 2007; 25: 1165-70.

31. Hardman R. A toxicologic review of quantum dots: toxicity depends on physicochemical and environmental factors. Environ Health Perspect. 2006; 114: 165-72.

32. Chan WC, Nie S. Quantum dot bioconjugates for ultrasensitive nonisotopic detection. Science. 1998; 281: 2016-8.

33. Yong KT, Qian J, Roy I, Lee HH, Bergey EJ, Tramposch KM, et al. Quantum rod bioconjugates as targeted probes for confocal and two-photon fluorescence imaging of cancer cells. Nano Lett. 2007; 7: 761-5.

34. Dubertret B, Skourides $\mathrm{P}$, Norris DJ, Noireaux $\mathrm{V}$, Brivanlou AH, Libchaber A. In vivo imaging of quantum dots encapsulated in phospholipid micelles. Science. 2002; 298: 1759-62.

35. Gao X, Cui Y, Levenson RM, Chung LW, Nie S. In vivo cancer targeting and imaging with semiconductor quantum dots. Nat Biotechnol. 2004; 22: 969-76.

36. Ling Y, Wei K, Luo Y, Gao X, Zhong S. Dual docetaxel/superparamagnetic iron oxide loaded nanoparticles for both targeting magnetic resonance imaging and cancer therapy. Biomaterials. 2011; 32: 7139-50.

37. Amstad E, Textor M, Reimhult E. Stabilization and functionalization of iron oxide nanoparticles for biomedical applications. Nanoscale. 2011; 3: 2819-43.

38. Louie A. Multimodality imaging probes: design and challenges. Chemical reviews. 2010; 110: 3146-95.

39. Lewinski N, Colvin V, Drezek R. Cytotoxicity of nanoparticles. Small. 2008; 4: 26-49.

40. Jung CW, Jacobs P. Physical and chemical properties of superparamagnetic iron oxide MR contrast agents: ferumoxides, ferumoxtran, ferumoxsil. Magn Reson Imaging. 1995; 13: 661-74. 
41. Nyk M, Kumar R, Ohulchanskyy TY, Bergey EJ, Prasad PN. High contrast in vitro and in vivo photoluminescence bioimaging using near infrared to near infrared up-conversion in $\mathrm{Tm} 3+$ and $\mathrm{Yb} 3+$ doped fluoride nanophosphors. Nano Lett. 2008; 8: 3834-8.

42. Zhou J, Sun Y, Du X, Xiong L, Hu H, Li F. Dual-modality in vivo imaging using rare-earth nanocrystals with near-infrared to near-infrared (NIR-to-NIR) upconversion luminescence and magnetic resonance properties. Biomaterials. 2010; 31: 3287-95.

43. Wang F, Banerjee D, Liu Y, Chen X, Liu X. Upconversion nanoparticles in biological labeling, imaging, and therapy. Analyst. 2010; 135: 1839-54.

44. Santra S. Fluorescent silica nanoparticles for cancer imaging. Methods Mol Biol. 2010; 624: 151-62.

45. Yang P, Gai S, Lin J. Functionalized mesoporous silica materials for controlled drug delivery. Chem Soc Rev. 2012; 41: 3679-98.

46. Dai Y, Ma P, Cheng Z, Kang X, Zhang X, Hou Z, et al. Up-conversion cell imaging and $\mathrm{pH}$-induced thermally controlled drug release from NaYF4/Yb3+/Er3+@hydrogel core-shell hybrid microspheres. ACS Nano. 2012; 6: 3327-38.

47. Gai S, Yang PY, Li CX, Wang WX, Dai YL, Niu N, et al. Synthesis of Magnetic, Up-Conversion Luminescent, and Mesoporous Core-Shell-Structured Nanocomposites as Drug Carriers. Adv Funct Mater. 2010; 20: 1166-72.

48. Longmire M, Choyke PL, Kobayashi H. Dendrimer-based contrast agents for molecular imaging. Curr Top Med Chem. 2008; 8: 1180-6.

49. Xu H, Regino CA, Koyama Y, Hama Y, Gunn AJ, Bernardo M, et al. Preparation and preliminary evaluation of a biotin-targeted, lectin-targeted dendrimer-based probe for dual-modality magnetic resonance and fluorescence imaging. Bioconjug Chem. 2007; 18: 1474-82.

50. Koyama Y, Talanov VS, Bernardo M, Hama Y, Regino CA, Brechbiel MW, et al. A dendrimer-based nanosized contrast agent dual-labeled for magnetic resonance and optical fluorescence imaging to localize the sentinel lymph node in mice. J Magn Reson Imaging. 2007; 25: 866-71.

51. Torchilin VP. Micellar nanocarriers: pharmaceutical perspectives. Pharm Res. 2007; 24: 1-16.

52. Torchilin VP. Structure and design of polymeric surfactant-based drug delivery systems. J Control Release. 2001; 73: 137-72.

53. Elbayoumi TA, Torchilin VP. Current trends in liposome research. Methods Mol Biol. 2010; 605: 1-27.

54. Puri A, Loomis K, Smith B, Lee JH, Yavlovich A, Heldman E, et al. Lipid-based nanoparticles as pharmaceutical drug carriers: from concepts to clinic. Crit Rev Ther Drug Carrier Syst. 2009; 26: 523-80.

55. Senior JH. Fate and behavior of liposomes in vivo: a review of controlling factors. Crit Rev Ther Drug Carrier Syst. 1987; 3: 123-93.

56. Laverman P, Carstens MG, Boerman OC, Dams ET, Oyen WJ, van Rooijen N, et al. Factors affecting the accelerated blood clearance of polyethylene glycol-liposomes upon repeated injection. J Pharmacol Exp Ther. 2001; 298: 607-12.

57. Litzinger DC, Buiting AM, van Rooijen N, Huang L. Effect of liposome size on the circulation time and intraorgan distribution of amphipathic poly(ethylene glycol)-containing liposomes. Biochim Biophys Acta. 1994; 1190: 99-107.

58. Allen TM, Hansen C. Pharmacokinetics of stealth versus conventional liposomes: effect of dose. Biochim Biophys Acta. 1991; 1068: 133-41.

59. Maham A, Tang $\mathrm{Z}, \mathrm{Wu} \mathrm{H}$, Wang J, Lin Y. Protein-based nanomedicine platforms for drug delivery. Small. 2009; 5: 1706-21.

60. Uchida M, Flenniken ML, Allen M, Willits DA, Crowley BE, Brumfield S, et al. Targeting of cancer cells with ferrimagnetic ferritin cage nanoparticles. J Am Chem Soc. 2006; 128: 16626-33.

61. Zhang L, Laug L, Munchgesang W, Pippel E, Gosele U, Brandsch M, et al. Reducing stress on cells with apoferritin-encapsulated platinum nanoparticles. Nano Lett. 2010; 10: 219-23.

62. Hosein HA, Strongin DR, Allen M, Douglas T. Iron and cobalt oxide and metallic nanoparticles prepared from ferritin. Langmuir. 2004; 20: 10283-7.

63. Ueno $\mathrm{T}$, Abe $\mathrm{M}$, Hirata $\mathrm{K}$, Abe $\mathrm{S}$, Suzuki M, Shimizu N, et al. Process of accumulation of metal ions on the interior surface of apo-ferritin: crystal structures of a series of apo-ferritins containing variable quantities of $\mathrm{Pd}(\mathrm{II})$ ions. J Am Chem Soc. 2009; 131: 5094-100.

64. Geninatti Crich S, Bussolati B, Tei L, Grange C, Esposito G, Lanzardo S, et al Magnetic resonance visualization of tumor angiogenesis by targeting neural cell adhesion molecules with the highly sensitive gadolinium-loaded apoferritin probe. Cancer Res. 2006; 66: 9196-201.

65. Santambrogio P, Levi S, Arosio P, Palagi L, Vecchio G, Lawson DM, et al. Evidence that a salt bridge in the light chain contributes to the physical stability difference between heavy and light human ferritins. J Biol Chem. 1992; 267: 14077-83.

66. Cai W, Chen K, Li ZB, Gambhir SS, Chen X. Dual-function probe for PET and near-infrared fluorescence imaging of tumor vasculature. J Nucl Med. 2007; 48: $1862-70$

67. Chen K, Li ZB, Wang H, Cai W, Chen X. Dual-modality optical and positron emission tomography imaging of vascular endothelial growth factor receptor on tumor vasculature using quantum dots. Eur J Nucl Med Mol Imaging. 2008; 35: $2235-44$.

68. Nahrendorf M, Keliher E, Marinelli B, Waterman P, Feruglio PF, Fexon L, et al. Hybrid PET-optical imaging using targeted probes. Proc Natl Acad Sci USA. 2010; 107: 7910-5.

69. Nahrendorf M, Waterman P, Thurber G, Groves K, Rajopadhye M, Panizzi P, et al. Hybrid in vivo FMT-CT imaging of protease activity in atherosclerosis with customized nanosensors. Arterioscler Thromb Vasc Biol. 2009; 29: 1444-51

70. Lin X, Xie J, Niu G, Zhang F, Gao H, Yang M, et al. Chimeric ferritin nanocages for multiple function loading and multimodal imaging. Nano Lett. 2011; 11: 814-9.

71. Zhang R, Lu W, Wen X, Huang M, Zhou M, Liang D, et al. Annexin A5-conjugated polymeric micelles for dual SPECT and optical detection of apoptosis. J Nucl Med. 2011; 52: 958-64.

72. Liang M, Liu X, Cheng D, Liu G, Dou S, Wang Y, et al. Multimodality nuclear and fluorescence tumor imaging in mice using a streptavidin nanoparticle. Bioconjug Chem. 2010; 21: 1385-8.

73. Lee HY, Li Z, Chen K, Hsu AR, Xu C, Xie J, et al. PET/MRI dual-modality tumor imaging using arginine-glycine-aspartic (RGD)-conjugated radiolabeled iron oxide nanoparticles. J Nucl Med. 2008; 49: 1371-9.

74. Choi JS, Park JC, Nah H, Woo S, Oh J, Kim KM, et al. A hybrid nanoparticle probe for dual-modality positron emission tomography and magnetic resonance imaging. Angew Chem Int Ed Engl. 2008; 47: 6259-62.

75. Misri R, Meier D, Yung AC, Kozlowski P, Hafeli UO. Development and evaluation of a dual-modality (MRI/SPECT) molecular imaging bioprobe. Nanomedicine. 2012; 8: 1007-16.

76. Misri R, Saatchi K, Ng SS, Kumar U, Hafeli UO. Evaluation of ${ }^{111}$ In labeled antibodies for SPECT imaging of mesothelin expressing tumors. Nucl Med Biol. 2011; 38: 885-96.

77. Park JC, Yu MK, An GI, Park SI, Oh J, Kim HJ, et al. Facile preparation of a hybrid nanoprobe for triple-modality optical/PET/MR imaging. Small. 2010; 6: 2863-8.

78. Joshi BP, Wang TD. Exogenous Molecular Probes for Targeted Imaging in Cancer: Focus on Multi-modal Imaging. Cancers. 2010; 2: 1251-87.

79. Xie J, Chen K, Huang J, Lee S, Wang J, Gao J, et al. PET/NIRF/MRI triple functional iron oxide nanoparticles. Biomaterials. 2010; 31: 3016-22.

80. Robertson R, Germanos MS, Li C, Mitchell GS, Cherry SR, Silva MD. Optical imaging of Cerenkov light generation from positron-emitting radiotracers. Phys Medicine Biol. 2009; 54: N355-65.

81. Lee J, Lee TS, Ryu J, Hong S, Kang M, Im K, et al. RGD peptide-conjugated multimodal $\mathrm{NaGdF}_{4}: \mathrm{Yb}^{3+} / \mathrm{Er}^{3+}$ nanophosphors for upconversion luminescence, MR, and PET imaging of tumor angiogenesis. J Nucl Med. 2013; 54: 96-103.

82. Hwang do $\mathrm{W}$, Ko HY, Lee JH, Kang $\mathrm{H}$, Ryu $\mathrm{SH}$, Song $\mathrm{IC}$, et al. A nucleolin-targeted multimodal nanoparticle imaging probe for tracking cancer cells using an aptamer. J Nucl Med. 2010; 51: 98-105.

83. Cherry SR. Multimodality in vivo imaging systems: twice the power or double the trouble? Annu Rev Biomed Eng. 2006; 8: 35-62.

84. Delbeke D, Schoder H, Martin WH, Wahl RL. Hybrid imaging (SPECT/CT and PET/CT): improving therapeutic decisions. Semin Nucl Med. 2009; 39: $308-40$.

85. Jennings LE, Long NJ. 'Two is better than one'--probes for dual-modality molecular imaging. Chem Commun (Camb). 2009;: 3511-24.

86. Lee S, Chen X. Dual-modality probes for in vivo molecular imaging. Mol Imaging. 2009; 8: 87-100.

87. Majumdar D, Peng $\mathrm{XH}$, Shin DM. The medicinal chemistry of theragnostics, multimodality imaging and applications of nanotechnology in cancer. Curr Top Med Chem. 2010; 10: 1211-26.

88. Shin SJ, Beech JR, Kelly KA. Targeted nanoparticles in imaging: paving the way for personalized medicine in the battle against cancer. Integr Biol-UK. 2013; 5: 29-42.

89. Cai H, Li Z, Huang CW, Shahinian AH, Wang H, Park R, et al. Evaluation of copper-64 labeled AmBaSar conjugated cyclic RGD peptide for improved microPET imaging of integrin alphavbeta3 expression. Bioconjug Chem. 2010; 21: $1417-24$

90. Cai H, Li Z, Huang CW, Park R, Shahinian AH, Conti PS. An improved synthesis and biological evaluation of a new cage-like bifunctional chelator, 4-((8-amino-3,6,10,13,16,19-hexaazabicyclo[6.6.6]icosane-1-ylamino)methyl)be nzoic acid, for ${ }^{64} \mathrm{Cu}$ radiopharmaceuticals. Nucl Med Biol. 2010; 37: 57-65.

91. Choi HS, Frangioni JV. Nanoparticles for biomedical imaging: fundamentals of clinical translation. Mol Imaging. 2010; 9: 291-310.

92. Kolosnjaj J, Szwarc H, Moussa F. Toxicity studies of carbon nanotubes. Adv Exp Med Biol. 2007; 620: 181-204.

93. Kolb HC, Sharpless KB. The growing impact of click chemistry on drug discovery. Drug Discov Today. 2003; 8: 1128-37.

94. Kolb HC, Finn MG, Sharpless KB. Click Chemistry: Diverse Chemical Function from a Few Good Reactions. Angew Chem Int Ed Engl. 2001; 40: 2004-21.

95. Zeng D, Lee NS, Liu Y, Zhou D, Dence CS, Wooley KL, et al. ${ }^{64} \mathrm{Cu}$ Core-labeled nanoparticles with high specific activity via metal-free click chemistry. ACS Nano. 2012; 6: 5209-19.

96. Chen K, Wang X, Lin W, Shen C, Yap L, Hughes LD, et al. Strain-Promoted Catalyst-Free Click Chemistry for Rapid Construction of ${ }^{64} \mathrm{Cu}$-Labeled PET Imaging Probes. ACS Med Chem Lett. 2012; 3: 1019-23.

97. Ahmed N, Fessi H, Elaissari A. Theranostic applications of nanoparticles in cancer. Drug Discov Today. 2012; 17: 928-34 